\title{
Magnetic Properties of Nanowires guided by Carbon Nanotubes
}

\author{
Miguel A. Correa-Duarte and Veronica Salgueirino \\ Universidade de Vigo \\ Spain
}

\section{Introduction}

The physical properties of one-dimensional (1D) nanostructures of magnetic materials are presently the subject of intensive research, taking into account the considerable attention they have recently received and the few cases reported. [1-4] Much of the early work was concerned with exploratory issues, such as establishing an easy axis for typical preparation conditions and the essential involvement of shape anisotropy, as opposed to magnetocrystalline anisotropy. More recently, attention has shifted towards the understanding of magnetization processes and related applications. Particularly interesting problems are the magnetic hysteresis of the wires and the time dependence of the magnetic reversal. Thus, magnetic nanowires have provided a highly successful test ground for understanding the microscopic mechanisms that determine macroscopically important parameters in the different applications where they can be used. [5]

On the other hand, these building blocks, as in the case of spherical nanoparticles, are at the border between the solid and molecular state displaying the novel effects that can now be exploited. Therefore, it becomes imperative to take into account the fact that the properties of materials composed of magnetic nanostructures are a result of both the intrinsic properties of the small building blocks and the interactions in between. [6]

This chapter is not meant as a survey of the present state and future developments of magnetic nanowires and since only two examples are considered, is far from being complete. The purpose of this chapter is three fold: a) an introductory level overview about magnetic colloids, the basic physics in the magnetism at the nanoscale; in terms of superparamagnetism, the concept of magnetic anisotropy and the dynamics of these systems. We have emphasized the dominant role of the surface effects on the intrinsic properties at the nanoscale and the competition with the interactions in the case of assemblies, leading to a characteristic magnetic behavior termed as spin-glass. Additionally, a brief introduction referred to carbon nanotubes (CNTs) is included. b) Characteristic examples of magnetic nanowires whose morphology was achieved by taking advantage of CNTs and exploiting wet-chemistry methods, and c) a complete analysis of the magnetic behavior displayed in both examples.

\subsection{Colloids}

Different preparation methods lead to magnetic nanostructures with differences in crystalline structure, surface chemistry, shape, etc. Hence, the fabrication technique has a

Source: Nanowires Science and Technology, Book edited by: Nicoleta Lupu, ISBN 978-953-7619-89-3, pp. 402, February 2010, INTECH, Croatia, downloaded from SCIYO.COM 
great influence on the magnetic properties of the materials obtained. Numerous physical and chemical methods have been employed to produce magnetic nanostructures, such as molecular beam epitaxy, chemical vapor deposition, normal incident pulsed laser deposition, sputtering or electrodeposition. The magnetic colloidal nanostructures or colloids are remarkably different if compared to nanostructures formed by these methods, as they are chemically synthesized using wet chemistry and are free-standing nanocrystals grown in solution. [7] The magnetic colloids are thus a subgroup of a broader class of the magnetic materials that can be synthesized at the nanoscale level but using wet chemical methods. In this fabrication of colloidal nanocrystals, the reaction chamber is a reactor containing a liquid mixture of compounds that control the nucleation and the growth. In general, each of the atomic species that will be part of the nanostructures is introduced into the reactor in the form of a precursor. A precursor is a molecule or a complex containing one or more atomic species required for growing the nanocrystals. Once the precursors are introduced into the reaction flask they decompose, forming new reactive species (the monomers) that will cause the nucleation and growth of the nanocrystals. The liquid in the reactor provides the energy required to decompose the precursors, either by thermal collisions or by a chemical reaction between the liquid medium and the precursors, or by a combination of these two mechanisms. [8] The key parameter in the controlled growth of colloidal nanocrystals is the presence of one or more molecular species in the reactor that we will term as ligands hereafter. A ligand is a molecule that is dynamically adsorbed to the surface of the growing structure under the reaction conditions. It must be mobile enough to provide access for the addition of monomer units, while stable enough to prevent the aggregation of nanocrystals in solution. Figure 1 provides a schematic illustration of a nanostructure with an example of a mobile ligand adsorbed on its surface. Due to the increased surface-to-volume ratio, surface chemistry is of great importance for the chemical and physical properties of the colloids likewise synthesized, generally being caused by these molecular ligands stabilizing them.

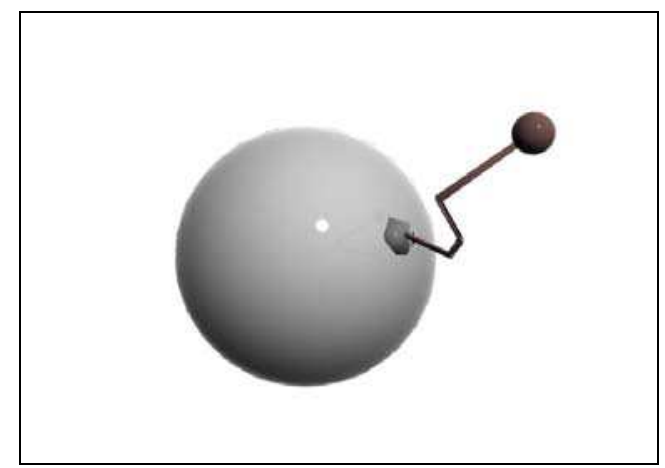

Fig. 1. Schematic illustration of a nanostructure with an example of a mobile ligand adsorbed on its surface.

The preparation of nanostructures of desired sizes is the first and very important step, being a prerequisite of their further investigation and use. Narrow size distribution or monodispersity is highly desirable because magnetic properties become strongly size dependent in the nanometer size range. Additionally, the magnetic behavior of nanostructures depends not only on their chemical composition and size, but also on their crystalline modification and the presence of structural defects like stacking faults or twinned 
planes. Consequently, Murray et al. proposed the use of the term "nanocrystal" for crystalline particles with low concentrations of defects, while the more general term "nanoparticle" also includes particles containing gross internal grain boundaries, fractures, or internal disorder. [9] Another important characteristic of the wet-chemistry methods refers to the fact that the colloids are dispersed in solution, and so that, they can be produced in large quantities in a reaction flask and can later be transferred to any desired substrate.

\subsection{Superparamagnetism}

It is well known that a magnetic body has a structure that is divided into uniformly magnetized regions (domains) separated by domain or Bloch walls. This distribution of regions or domains permits the magnetic body to minimize its magnetostatic energy. Since it is the total energy that requires to be minimized, it will be a final balance of energies (including the magnetostatic and the exchange terms, the different anisotropies that come into play and the domain walls contribution) what will determines the domain structure. In order to work at the nanoscale, there will be an important reduction in dimensions, so that, the size of the domains will also be reduced. At this point, the domain structure will consequently change, but due to the domain wall formation energy cost, the balance with the magnetostatic energy will limit the subdivision in domains to a certain optimum size. Consequently, there is a size limit below which the nanostructure can no longer gain an energy favorable configuration by breaking up into domains. Hence, it remains with only one domain. The critical size, or single domain size $D_{s}$, below which a particle will not form domains, is where these two energies (energy cost of domain walls formation and magnetostatic energy) become equal, and the typical values for $D_{s}$ range from 10 to $100 \mathrm{~nm}$, with elongated nanostructures tending to have larger $D s$.

Much of the behavior of these single-domain magnetic nanostructures can be described by assuming that all the atomic moments are rigidly aligned as a single "giant" spin. This is in essence the theory of superparamagnetism. Below the Curie temperature of a ferromagnet or ferrimagnet, all the spins are coupled together and so cooperate to yield a large total moment $\mu$ (the giant spin). This moment is bound rigidly to the nanostructure by one or more of the variety of anisotropies (that will be later discussed). The energy of this bond is $K V$, where $K$ is the effective magnetic anisotropy (that takes into account the variety of anisotropies that come into play) and $V$ is the volume of the particle. With decreasing size, the $K V$ magnitude decreases and the thermal energy $k T$ can disrupt the bonding of the total magnetic moment to the nanostructures. The magnetic moment in this situation is free to move and respond to an applied field independently of the nanostructure itself. An applied field would tend to align it, but $k T$ would fight the alignment just as it does in a paramagnet (justifying therefore the use of the term superparamagnet). [10]

The phenomenon of superparamagnetism is, in fact, timescale-dependent due to the stochastic nature of the thermal energy $k T$. The anisotropy energy $K V$ represents an energy barrier to the total spin reorientation, hence the probability for jumping this barrier is proportional to the Boltzmann factor $\exp (-K V / k T)$. This can be made quantitative by introducing an attempt timescale, $\tau_{0}$ which describes the timescale over which $\mu$ attempts to jump the $K V$ barrier. Then, the timescale for a successful jump is

$$
\tau=\tau_{0} e^{-K V / k T}
$$


The attempt timescale is about $10^{-9} \mathrm{~s}$. The typical experiment with a magnetometer takes 10 to $100 \mathrm{~s}$; and if $M_{S}$ reverses at times shorter than the experimental timescale, the system appears superparamagnetic. Thus, using $\tau \approx 100 \mathrm{~s}$ and $\tau_{0}=10^{-9} \mathrm{~s}$, the critical volume becomes:

$$
\begin{gathered}
V_{s p}=\frac{25 k T}{K} \\
T_{B}=\frac{K V}{25 k}
\end{gathered}
$$

The equation (2) can be rearranged to yield equation (3) and gives the blocking temperature $T_{B}$. Below $T_{B}$ the free movement of $\mu=M_{S} V$ is blocked by the anisotropy; above $T_{B}, k T$ permits the magnetic moment $(\mu)$ to fluctuate freely, so that the system appears superparamagnetic.

\subsection{Magnetic anisotropy}

The magnetic anisotropy concept describes the fact that the energy of the ground state of a magnetic system depends on the direction of the magnetization. The effect occurs either by rotations of the magnetic moment $\mu$ with respect to the external shape of the specimen (shape anisotropy) or by rotations relative to the crystallographic axes (intrinsic or magnetocrystalline anisotropy). The direction(s) with minimum energy, i.e. into which the magnetic moment $\mu$ points in the absence of external fields are called easy directions. The direction(s) with maximum energy are called hard directions. The magnetic anisotropy energy (MAE) between two crystallographic directions is given by the work needed to rotate the magnetic moment $\mu$ from an easy direction into the other directions. The MAE, that corresponds to a small contribution to the total energy of a bulk crystal, becomes more and more important as decreasing the size of the magnetic material until reaching the nanoscale, depending on different issues.

In bulk materials, magnetocrystalline and magnetostatic energies are the main sources of anisotropy, but in nanosized structures, other types of anisotropy can be of the same order of magnitude. As the properties are stated by the relaxation time $\tau$ of the magnetic moment $\mu$ on the nanostructures, $\tau$ being itself governed by the energy barrier $K V$ (directly dependent on the effective magnetic anisotropy $K$ ), it is important to know all the possible sources of anisotropies and their contribution to the total energy barrier.

There are fundamentally two sources of magnetic anisotropy; (i) spin-orbit (LS) interaction and (ii) the magnetic dipole-dipole interaction. The more important interaction is the spinorbit coupling, which couples the spin to the charge (orbital) density distribution in the crystal. Thus, the magnetic moment $\mu$ "gets the feel" of the crystal via the orbital motion of the magnetic electrons. The orbital motion is coupled to the lattice by an electric field (that indeed reflects the symmetry of this lattice). This field $K$ is given by the sum of the electrostatic potentials $\phi\left(r_{i}\right)$ over the nearest neighbors at sites $r_{i}$. [11]

$$
K=\sum_{i}^{N} e \cdot \phi\left(\left|\vec{r}_{i}\right|\right)
$$


The first source (spin-orbit) of magnetic anisotropy includes the so-called magnetocrystalline and magneto-elastic contributions while the second one (from magnetic dipoledipole interactions) includes contributions termed shape or magneto-static anisotropy. [12] Accordingly, when considering the nanoparticulate systems, there are main contributions referred to the magnetocrystalline anisotropy whose energy can show various symmetries (uniaxial and cubic forms cover the majority of the cases), to the surface anisotropy (related to surface effects that stem from the fact that the existence of the surface represents a discontinuity for magnetic interactions) and to magnetostatic energy. Additionally, stress anisotropy should be taken into account as well since there is a second effect due to the surface, related to strains, because of a magnetostriction effect. [13]

If we refer to the magnetocrystalline anisotropy, the direction of the spontaneous magnetization of crystalline samples is oriented along certain directions. Typically, for bulk samples of bcc-Fe, fcc-Ni, and hcp-Co, these are the cubic $\langle 100\rangle,\langle 111\rangle$ and hexagonal $\langle 0001\rangle$ directions, respectively. These directions are called easy magnetization directions and the magnetization along any other direction requires an excess energy. [14] The magnetic anisotropy energy density is indeed the excess work that needs to be put into the system to achieve saturation magnetization along a non-easy axis of magnetization. This excess work depends on the orientation of the magnetizing field with respect to the sample, and its magnitude will be different in general for magnetization in the different directions of the crystalline lattice. In sharp contrast to the small crystalline anisotropy of bulk samples, ultrathin films and nanostructures often exhibit an effective magnetic anisotropy that is orders of magnitude larger than the respective bulk value. The deviation from the respective bulk values has been ascribed in most cases to a magnetocrystalline surface anisotropy of the Néel type. [15]

In general, one can always apply the rule that indicates that the lower the symmetry of the crystals or of the local electrostatic potential around the magnetic moment, the larger the MAE is. [16] The mentioned increase of magnetic anisotropy for ultrathin films and nanostructures is often ascribed to so-called interface and surface anisotropy contributions that are attributed to the different atomic environment of the interface and surface atoms, where the symmetry of the crystals is much lower than in internal positions. [12]

The important impact of symmetry on the resulting magnetic anisotropy can also be understood in the crystal field description, which has been applied successfully to describe the magnetic anisotropy of $3 \mathrm{~d}$ ions. [17] The energetically degenerate $3 \mathrm{~d}$ levels of a free atom split into two groups, $e_{g}\left(d_{z} 2, d_{x} 2-y 2\right.$ orbitals $)$ and $t_{2 g}\left(d_{x y}, d_{x z}\right.$ and $d_{y z}$ orbitals $)$ in the presence of the electric field of the surrounding atoms. The energy separation and the relative positioning of the $e_{g}$ and $t_{2 g}$ levels depend on the symmetry of the atomic arrangement [18] and a tetragonal distortion lifts the degeneracy of these levels. [19] Thus, a change of symmetry, e.g. induced by strain, may lead to a change of the relative occupancy of the different d-orbitals, which in turn leads via spin-orbit coupling to a change of the magnetic anisotropy.

In order to illustrate the role of the symmetry (and surface effects) we can consider the following case. At each lattice point within for example, a cubic lattice, the dipole fields of all neighbors cancel out. This is, however, only true for an infinite system. As soon as surfaces are present, magnetic poles develop and thus, the dipole-dipole interaction leads to anisotropy. Since the shape of the sample determines this anisotropy (even for modest shape ratios $c / a$, the shape anisotropy can be very large), one usually calls it shape anisotropy. As a 
result, in systems with reduced dimensions as nanostructures, the shape anisotropy might be even the dominating contribution to the overall MAE. [16]

Another important contribution comes from a well-known experimental fact derived from the coupling between magnetism and lattice strain, the magnetostriction, which origins the magnetoelastic anisotropy. A sample lowers its total energy upon magnetization by a lattice strain that depends on the magnetization direction with respect to the crystalline lattice. The underlying principle of the so-called magnetoelastic coupling can be described as the strain dependence of the magnetic anisotropy energy density. [20] For thin films and nanostructures, since are generally under considerable strain, this contribution can also determine the magnetic anisotropy.

Cantilever bending experiments and nanoindentation techniques are sensitive and accurate tools for the measurement of mechanical stress in atomic layers, at surfaces and on nanoparticles. The idea of the measurement in the former case is to detect the stress-induced change in curvature of a thin substrate. The curvature change is directly proportional to the film stress, integrated over the film thickness. The second case, the nanoindentation, has now long been used to study the elastic, plastic, and fracture properties of surfaces of bulk samples, and in the last decade, it has become possible to perform controlled compression and bending tests on nanostructures smaller than a micron, such as NPs, [20-24] nanowires [25-26] and nano-pillars. [27-28] In both cases, the elastic properties of the magnetic structures are given by the Young's modulus and the Poisson ratio and the magnetoelastic coupling coefficients can then be derived.

Although the important aspect of magnetic domain formation is not discussed here since we have restricted the scope of the introduction to theoretical single-domain nanostructures behavior, we will actually take into account the interplay of magnetostatic energy, exchange energy and the effective magnetic anisotropy that can lead to an energetically favored multidomain state. Hence, aspects of configurational magnetic anisotropy that appear in submicron-sized magnets due to small deviations from the uniform state [29] and the socalled exchange anisotropy are considered.

The phenomenon of exchange anisotropy is the result of an interfacial exchange interaction between ferromagnetic (FM) and antiferromagnetic (AFM) materials, and only recently have the required experimental and analytical tools for dealing with interfacial behavior at the atomic level become available. [30] The observation (below room temperature) of a hysteresis loop shifted along the field axis, after cooling a nanostructured system in an applied field, indicates an exchange interaction across the interface between a FM and an AFM materials composing the sample. This loop shift was demonstrated to be equivalent to the assumption of a unidirectional anisotropy energy in the expression for the free energy at $T=0 K$ of single-domain spherical particles with uniaxial anisotropy, aligned with their easy axis in the direction of the field, $H$, which is applied anti-parallel to any particle's magnetization $M_{s}$ :

$$
F=H M_{s} \cos \theta-K_{u} \cos \theta+K_{1} \sin ^{2} \theta
$$

where $\theta$ is the angle between the easy direction and the direction of magnetization, and $K_{u}$ and $K_{1}$ are the unidirectional and uniaxial anisotropy energy constants, respectively. Solutions of this equation are readily expressed in terms of an effective field $H^{\prime}$,

$$
H^{\prime}=H-\frac{K_{u}}{M_{s}}
$$


that offers the hysteresis loop displaced by $K_{u} / M_{s}$, on the $H$-axis. Thus, an explanation of the loop shift is equivalent to explaining the unidirectional anisotropy.

AFM materials appear magnetically ordered below their Néel temperatures $T_{N}$, and have a zero net moment since have parallel and anti-parallel spins in a preferred direction. However, at the interface with a FM, there are localized net moments that arise from several sources. Indeed, in AFM nanostructures with compensated interfacial spin planes, there can be unequal numbers of parallel and antiparallel spins at the surface of the nanostructure, due to various origins such as its size, shape or roughness, generating localized net AFM moments. Since the FM is ordered at its Curie temperature $T_{C}$, greater than the $T_{N}$ of the AFM, a field applied to couple FM-AFM systems at $T>T_{N}$ will align the FM magnetization in the field direction, while the AFM spins remain paramagnetic. As the temperature is lowered through $T_{N}$, the ordering net localized AFM spins will couple to the aligned FM spins, sharing their general spin direction. For high AFM magnetocrystalline anisotropy, if the interfacial AFM spins are strongly coupled to the AFM lattice, they will not be substantially rotated out of their alignment direction by fields applied at temperatures below $T_{N}$. However, since the localized uncompensated AFM spins are coupled to FM spins at the interface, they exert a strong torque on these FM spins, tending to keep them aligned in the direction of the cooling field, i.e., a unidirectional anisotropy.

Skumryev et al. demonstrated a magnetic coupling of FM nanoparticles with an AFM matrix as a source of a large effective additional anisotropy that led to a marked improvement in the thermal stability of the moments of the FM nanoparticles. The mechanism provides a way to beat the "superparamagnetic limit" in isolated particles so that, with the right choice of FM and AFM components, exchange anisotropy coupling could ultimately allow magnetically stable nanostructures. These nanostructures, only a few nanometers in size, would be able to surpass the storage-density goal of $1 \mathrm{Tbit}^{\mathrm{in}} \mathrm{N}^{-2}$, as set by the magnetic storage industry. [31]

As stated, the surface anisotropy is expected to contribute decisively for systems dominated by their surface properties, e.g. nanostructures offering an increased surface/volume ratio as it is the case of the magnetic colloids discussed here. Indeed, when it comes to magnetic nanostructures, the dominant surface contributions stem from the decisive role of ligands coverage onto the surface of the nanoparticles in order to render them dispersable in different solvents (colloidal chemistry), and therefore, became relevant for the resulting magnetic anisotropy. Experimental and theoretical reports have identified this decisive role of the ligands or molecular compounds adsorbed on the surface of different nanostructures in their magnetic anisotropy. [32] Since small structural relaxations can be induced by these ligands due to an induced spin reorientation related to the magnetoelastic coupling, it can be concluded that this magnetoelastic coupling opens the way to influence the magnetic anisotropy by even subtle structural and chemical changes at the surface. [33-36] Thus, surface magnetic properties result basically from the breaking of symmetry of the lattice, $[37,38]$ which leads to site-specific, generally uniaxial, surface anisotropy, and from broken exchange bonds, which inevitably lead to surface spin disorder and frustration (most prominently in oxydic ferro-, antiferro- and ferrimagnets). [39,40]

On the other hand, the coordination of the ligands to the nanostructures surface should not alter the intrinsic specific physical properties of the particles nor those induced by their nanometer size. This latter point is important in order to take advantage of both the intrinsic and collective properties for future applications. Dumestre et al. have reported an 
organometallic route towards the synthesis of metallic nanoparticles that can be applied to magnetic ones, based on the decomposition of an olefinic complex under a controlled atmosphere of $\mathrm{H}_{2}$ in mild conditions of pressure. [41] Respaud et al. were able to demonstrate that the surface of this type of nanoparticles is free of contaminating agents so that, the magnetic properties are identical to those observed for nanoparticles produced and studied in ultra high vacuum. [42]

The magnetic anisotropy of magnetic nanostructures can therefore be tuned by a proper selection of the combination between the magnetic material itself and the molecular ligands attached to their surface to stabilize them, and by adjusting the growth parameters in the synthetic process used (herein we refer specifically to colloidal chemistry methods). Thus, the link between growth parameters and magnetism is given by the correlation between the nanostructures morphology, structure and the magnetic properties themselves. This correlation is given by the magnetoelastic coupling, and by the length scale of structure and morphological variations as compared to the length scale that describes magnetic properties. Such a magnetic length scale is for example, the domain wall, the exchange stiffness or the magnetic anisotropy. [12]

Additionally, despite the fact that we have just introduced single-domain nanostructures, if we consider the magnetization reversal by domain motion, an impact of structural and magnetic anisotropy variations on the coercivity will be expected. These variations should occur on a length scale larger than the domain wall width otherwise the magnetic inhomogeneities will be average out by the magnetic exchange interactions, and only little impact will result on the coercivity. [43]

\subsection{Collective magnetic behavior}

One of the most attractive topics in the field of condensed matter physics is slow dynamics such as nonexponential relaxation, aging (a waiting time dependence of observables) and memory effects. In the field of spin glasses, slow dynamics has been studied widely both experimentally and theoretically to examine the validity of novel concepts, such as a hierarchical organization of states and temperature chaos. These extensive studies have revealed interesting effects like memory and rejuvenation $[44,45]$ that led to also study slow dynamics in dense magnetic nanoparticulate systems by using experimental protocols already developed.

In the case of magnetic nanostructures systems, there are two possible origins of slow dynamics. The first one is a broad distribution of relaxation times originating solely from that of the anisotropy energy barriers of each nanoparticle moment. This is the only source of slow dynamics for sparse (weakly interacting) magnetic nanoparticle systems, in which the nanoparticles are fixed in space. However, for dense magnetic nanoparticle systems, there is a second possible origin of slow dynamics, namely, cooperative spin-glass dynamics due to frustration caused by strong dipolar interactions among the particles and randomness in the particle positions and anisotropy axis orientations. [46, 47] The 3D random distributions and random orientations of anisotropy axes of such nanostructures in an insulating matrix with high enough packing density and sufficiently narrow size distribution will create a competition of different spin alignments. [48] Which of the two is relevant depends essentially on the concentration of the particles. Therefore, in order to understand appropriately slow dynamics in magnetic nanoparticle systems, it is desirable to clarify both. 
The comparison of the phenomena observed in relation to slow dynamics reveals some properties peculiar only to spin glasses, e.g. the flatness of the field-cooled magnetization below the critical temperature and memory effects in the zero-field-cooled magnetization. These two effects reflect the instability of the spin-glass phase under a static magnetic field of any strength meaning that it is indeed far from equilibrium. [49]

The intriguing properties of magnetic nanostructures including superparamagnetic and spin glass behavior are often ascribed to the delicate interplay between intrinsic properties and magnetostatic interactions.[50] When comparing between superparamagnets and spin-glasslike behavior of nanostructures, one significant difference is seen in the $M_{F C}$ without intermittent stops (when cooling the system). While in the case of superparamagnets this $M_{F C}$ increases as decreasing temperature, in the second case, the nearly constant $M_{F C}$ is actually considered to be a typical property of ordinary spin glasses. Additionally, a further important phenomenon that is peculiar to superspin-glasses is the memory effect in the genuine ZFC protocol. This memory experiment in a ZFC protocol permits to differentiate between spin-glass phase and superparamagnets interacting. In this experiment, $M_{Z F C}$ is measured with and without stops and in both cases cooling (and reheating) rate has to be the same. The stopping temperatures must be well below the blocking temperature $T_{B}$ of the system measured. The results of the experiment will give us no significant difference in $M_{Z F C}$ at the stopping temperatures and below when considering magnetic nanostructures weakly interacting. In this case then, the origin of slow dynamics is without doubt the broad distribution of relaxation times originating solely from the distribution of magnetic anisotropy energies of the nanostructures considered. On the contrary, if there is a significant difference in $M_{Z F C}$ at the stopping temperatures and below, we must now considered our system of magnetic nanostructures as offering a spin-glass-like behavior.

It is important to carry out this latter experiment considering the $M_{Z F C}$ and not the $M_{F C}$ since only a spin glass left unperturbed by external fields at constant $T$, rearranges its spin configuration through a very slow process to reduce the domain wall energy. [51] Indeed, all measurements should be done in quite low magnetic fields in order to avoid nonlinear effects. ${ }^{48}$ Moreover, Jonsson et al. reported that the collective behavior due to dipole-dipole interactions in concentrated samples extended the magnetic relaxation towards longer times and at the same time suppressed the relaxation at short observation times, a behavior that conforms to characteristic spin glass dynamics. [52]

One more point to be concerned about is the fact that collective magnetic excitations account for the precession or oscillations of the magnetic moment in the nanostructure about its magnetic easy axis, triggered at low temperatures by thermal energies insufficient to induce spin flips between opposite directions of the anisotropy axis, for $k_{B} T<K_{\text {eff }} V$. However, the particle surface-energy landscape can accommodate additional local minima due to lower coordination of the surface atoms, surface strain and spin canting associated with the surface itself. Many studies indicate that the breakage of superexchange bonds results in the creation of a surface shell within which spin disorder leads to spin-glass-like phase at the surface with closely spaced equilibrium states. [39, 53]

\subsection{Carbon nanotubes}

A great example representing the ever-increasing nanoscale-based research refers to the $\mathrm{sp}^{2-}$ bonded carbon nanotubes (CNTs) discovered in 1991. [54] CNTs have been pointed out as a paradigm material when talking about Nanotechnology. This new form in the carbon family 
with remarkable structure-dependent electronic, mechanical, optical and magnetic properties [55] has been the focus of an intensive study directed to numerous applications on many different fields, [56,57] including synthetic routes where CNTs are useful for biological applications. [58,59] Therefore, CNTs are expected to be controllably assembled into designed architectures as integral components of composites and/or supramolecular structures.

CNTs are hollow cylinders consisting of single or multiple sheets of wrapped graphite. According with the number of layers they are classified as single-walled carbon nanotubes (SWNTs) or multi-walled carbon nanotubes (MWNTs). Usually the structure of CNTs can be characterized using a pair of integers $(n$ and $m$ ), which give us the rolling-up direction of the carbon sheet and the nanotube diameter. Thus, depending on the different rolling-up modes, the CNTs can be named as armchair with $n=m$, zigzag with $n=0$ or $m=0$, or generally chiral when any other $n$ and $m$. Many of the potential applications of SWNTs and MWNTs are highly dependent on their electronic properties and in this context, their ballistic transport behavior and long electron mean free path have shown their potential as molecular wires. [60]

On the other hand, CNTs exhibit excellent structural flexibility and fluidity and can be bent, collapsed, or deformed into various shapes such as buckles, rings, or fullerene onions, providing a variety of shape-controlled physical properties of the nanostructures. Also by varying the geometric structure of the CNTs one can control electronic properties such as electrical conductivity or electron emission properties, thus providing modified electronic characteristics of the functional devices based on the CNTs.

Organic compounds have been used as templates for the generation of inorganic, organic or biological structures and materials rendering themselves the object of an increasing interest over the last years. In this way, taking into account that CNTs have been synthesized as an array of unprecedented structural, mechanical, and electronic properties, together with their high aspect ratio and surface area, these features have pointed them out as ideal templates for the deposition of a number of different materials on the search of new composite structures with promising properties and applications, offering the structural support that most of the inorganic, organic or biological materials generally lack. [61,62]

This ability to shape materials on a microscopic level is always desired but usually deficient. The formation of these hybrid structures is challenging due to the great application potential they display in many different fields and also from the scientific viewpoint. The application of these materials in useful processes and devices is ensured as soon as their production will be accomplished in a precise, reproducible manner, and if possible at reasonable costs.

Most of the applications proposed for CNTs have been shown to be strongly dependent on the development of strategies for functionalizing, processing and/or assembly of the CNTs themselves, mainly because their surface is rather inert, rendering very difficult any type of the mentioned mechanisms or techniques.

This is the case of a material deposition process onto CNTs, since becomes very difficult to control the final homogeneity. It is therefore important to explore feasible techniques whereby a surface modification would guarantee this material deposition onto the surface of the CNTs as a prior functionalization. Several approaches for functionalization of CNTs that have been developed can be classified as defect-site chemistry, covalent side-wall functionalization and non-covalent functionalization (figure 2). 


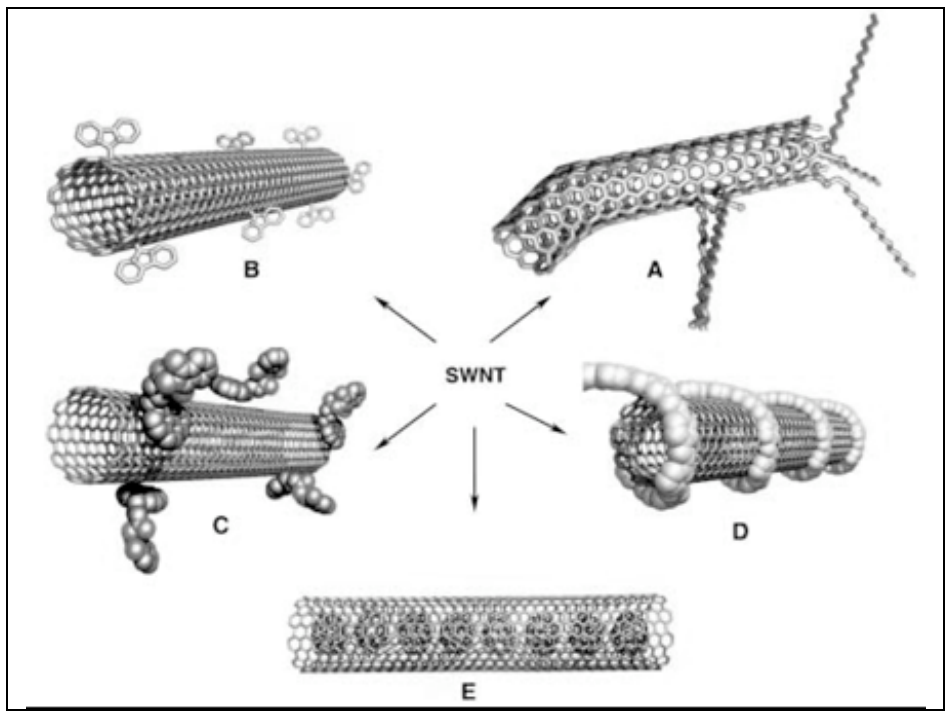

Fig. 2. Different types of SWNTs funtionalization: defect-site functionalization (A), covalent sidewall functionalization (B), non-covalent functionalization through, surfactants (C) or wrapping with polymers (D), and endohedral functionalization (E). Reprinted with permission from reference number 64. Copyright $2002 \mathrm{Wiley-VCH}$.

Defect-site chemistry Defect-side chemistry exploits the intrinsic defect sites both at the end and on the sidewalls of the CNTs as a result of the synthetic process. Additionally, the purification process of the CNTs involves the use of strong acids to remove the catalytic particles necessary for the synthesis, due to their oxidative action. This process ends up in holes with oxygenated functional groups like carboxylic acids or alcohol groups among others, which are promising starting points for the attachment of particles, molecular moieties or for further coordination chemistry at these sites.

Covalent-sidewall functionalization Covalent-sidewall functionalization is based on the chemical reactivity of the CNTs, related with the pyramidalization of the sp²-hybridized carbon atoms and the $\Pi$-orbital misalignment between adjacent carbon atoms. This pyramidalization and misalignment are scaled inversely with tube diameter, becoming more reactive tubes as decreasing their diameter. This agrees with the fact that fullerenes have a higher reactivity surface (which depends strongly on their curvature) compared to SWNTs which have no strongly curved regions that could serve for direct additions. This statement also explains why side-functionalization of SWNTs by covalent-bond formation needs highly reactive reagent.

Non-covalent functionalization Non-covalent functionalization comprises the dispersion of CNTs in aqueous solution, by means of surface active molecules as sodium dodecylsulfate (SDS) or by wrapping them with polymers. While the first one accommodates the CNTs in their hydrophobic interiors (sometimes by strong $\Pi-\Pi$-stacking interactions with the CNT sidewall if the hydrophobic part contains an aromatic group), the second one implies an association of the polymers with the sides of the CNTs based on the hydrophobic thermodynamic preference of CNT-polymer interactions compared to CNT-water interactions, thereby suppressing the hydrophobic surface of the CNTs. 
Endohedral functionalization Endohedral functionalization comprises the use of the inner cavity of the CNTs for the storage of molecules or compounds since their interaction takes place with the inner surface of the sidewalls, very convenient for confined reactions inside the CNTs.

In summary, defect-side functionalization preserves the electronic structure of the CNTs, since the nanotubes can tolerate a number of defects before losing their unique electronic and mechanical properties. Covalent-sidewall functionalization generates a high degree of functionalization rendering this method very useful for composites formation. However, the destruction of the $\mathrm{sp}^{2}$-hybridized structure may result in a loss of thermal conductivity, reducing the maximum buckling force or changing their electronic properties, displaying a semiconductor instead of a metallic behaviour.[63] Finally, the non-covalent functionalization has the main advantage since preserves intact the electronic properties and structure of the CNTs by maintaining the intrinsic nanotube sp2-hybridization. [64]

\section{Nanowires magnetic properties guided by carbon nanotubes}

Several methods have been exploited for the synthesis of magnetic colloids on which the magnetic anisotropy can be tuned; relatively simple variations in surfactant composition used to selectively control the growth rates of different faces (in similar procedures as those concerning semiconductor materials $[65,66]),[1]$ assembling previously synthesized magnetic nanoparticles as chains or necklaces, [2-4] [67-69] exploiting electrostatic interactions between the surface charge of magnetic nanoparticles and previously modified carbon nanotubes (CNTs), [70] or depositing the magnetic material on the surface of CNTs in a stepby-step procedure, giving place to homogeneous outer shells.[71] In order to investigate the possibility of obtaining nanowires with a very narrow size distribution and without chemical bonding at the surface we have chosen the third and fourth options schematically illustrated in figure 3. Thus, we have recently demonstrated that driving iron oxide nanoparticles or the direct reduction of nickel salt onto the surface of CNTs (in the second case in the presence of $\mathrm{Pt}$ nanoparticles) leads to very homogeneous magnetic nanowires.

\subsection{Synthetic strategies}

a. $\mathrm{Fe}_{3} \mathrm{O}_{4} / \gamma$ - $\mathrm{Fe}_{2} \mathrm{O}_{3}$-coated CNTs

For this first strategy designed, an assembly of a compact layer of magnetic nanoparticles onto CNTs was taken under consideration. This option was carried out following a procedure that combines the polymer wrapping and LbL self-assembly techniques allowing the non-covalent attachment of iron oxide nanoparticles onto the CNTs and thus leaving intact their structure and electronic properties (see figure 2, D functionalization of CNTs by the polymer wrapping). [72] Poly (Sodium 4-styrene sulfonate) (PSS) was used as the initial wrapping polymer that permits to provide remarkably stable aqueous dispersions of multiwall carbon nanotubes (MWNTs). Due to the high density of sulfonate groups on this negatively charged polyelectrolyte, the PSS acts as a primer onto the CNTs surface. So that, the subsequent and homogeneous absorption through electrostatic interactions of a cationic polyelectrolyte, the poly-(dimethyldiallylammonium chloride) (PDDA) is possible, supplying a homogeneous distribution of positive charges. These positive charges ensure the efficient adsorption (exploiting the same phenomenon, by means again of electrostatic interactions) of negatively charged magnetic nanoparticles onto the surface of CNTs. 
Although the adsorption of nanoparticles (diameter D $=6-10 \mathrm{~nm}$ ) onto CNTs is often problematic due to the extremely high curvature $\left(\mathrm{D}_{\mathrm{MWCNT}}=15-30 \mathrm{~nm}\right)$ that hinders the formation of dense coatings, the LbL self-assembly technique overcomes these difficulties, basically because of the electrostatic nature of the responsible interactions. [73, 74]

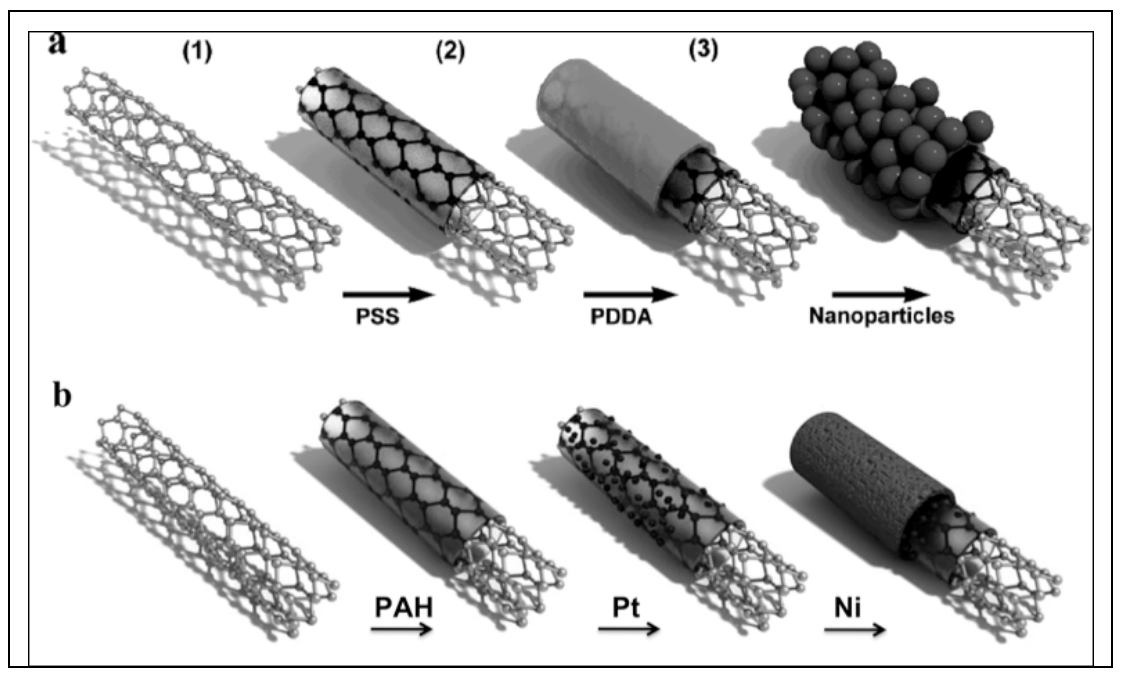

Fig. 3. Schematic illustrations of the synthetic processes used to produce magnetic nanowires using CNTs as guides and supports; a) by driven iron oxide nanoparticles and b) by reducing $\mathrm{Ni}$ atoms in a step-by-step process helped by the presence of catalytic Pt sites.

The magnetic nanoparticles prepared in aqueous solution (basic $\mathrm{pH}$ ) are as mentioned, negatively charged and therefore electrostatically attracted to the positively charged PDDA outer layer adsorbed onto the CNTs. The $\mathrm{pH}$ for the most efficient adsorption of the $\mathrm{Fe}_{3} \mathrm{O}_{4} / \gamma-\mathrm{Fe}_{2} \mathrm{O}_{3}$ nanoparticles on polyelectrolyte was found to be 11.9-12.0 while at higher $\mathrm{pH}$ values no adsorption was observed. A uniform coating of magnetic particles onto the CNTs was achieved, as shown in the TEM images of Figure 4 (a and b) where long ( $>5 \mu \mathrm{m})$ CNTs appeared completely covered with a dense layer of magnetic nanoparticles. These aqueous dispersions of magnetite/maghemite-coated CNTs were found to be very stable for several days or even weeks in the case of dilute solutions when (after centrifugation/washing) $\mathrm{TMAOH}$ (tetramethyl ammonium hydroxide) was added to the solution. Since the early work by Massart, [75] TMAOH has been a popular stabilizer for the preparation of aqueous ferrofluids, mainly comprising iron oxide nanoparticles. The stabilization of these magnetic CNTs in water takes place analogously through the formation of a double layer, with negative hydroxide ions fixed on the surface of the magnetic composites and positive tetramethylammonium as counterions in the basic environment.

Once coated, the magnetic response of these CNTs-based composites was easily and quickly visualized by holding the sample close to a small magnet. Indeed, when a drop of the dispersion was dried on a Si wafer without an external field, the magnetic CNTs were found randomly oriented on the silicon substrate (figure 5a). However, the magnetic CNTs can be oriented in the plane of a silicon wafer by using the mentioned external magnetic field. The magnetite/maghemite-coated CNTs were aligned as long chain structures by means of a 


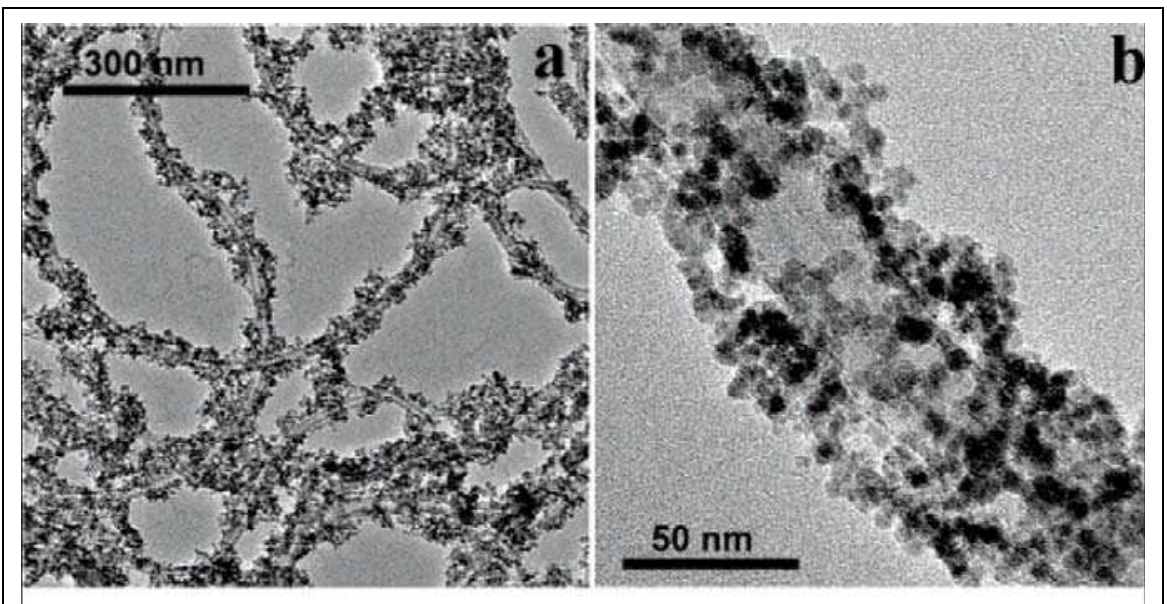

Fig. 4. TEM images at lower (a) and higher (b) magnifications of iron oxide-coated CNTs.
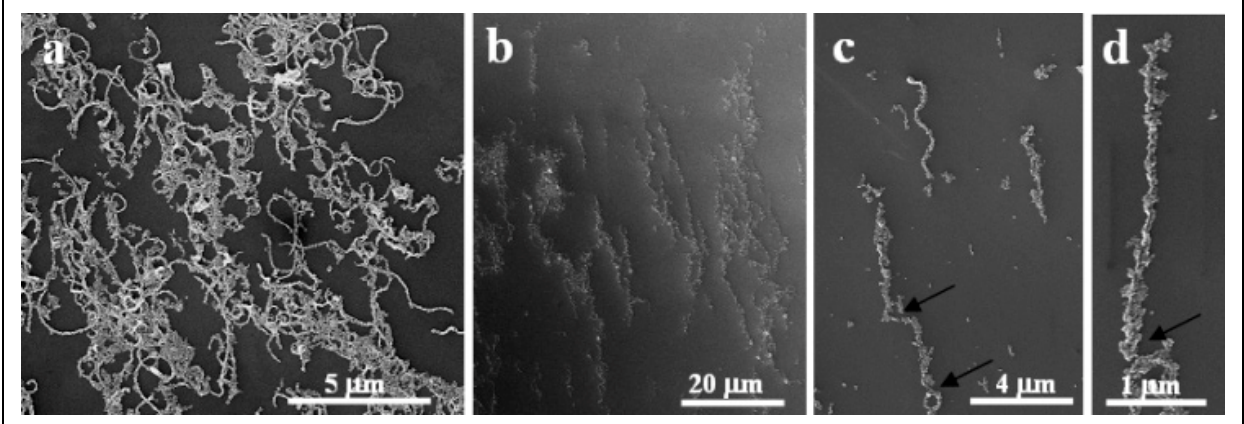

Fig. 5. SEM images of $\mathrm{Fe}_{3} \mathrm{O}_{4}$-coated CNTs in the absence (a) and in the presence (b,c,d) of an external magnetic field.

magnetophoretic deposition process on the silicon substrate at $300 \mathrm{~K}$, since magnetic CNTs suspended in a liquid align parallel to the direction of the applied magnetic field (in this case under a magnetic field of $0.2 \mathrm{~T}$ ). This fact becomes quite easy to understand as considering that in zero field, the magnetic moments of the iron oxide nanoparticles randomly point in different directions resulting in a vanishing net magnetization (due to the thermal fluctuations able to influence the magnetic moments of the nanoparticle at this temperature). However, if a sufficiently large homogeneous magnetic field is applied, the magnetic moments of the nanoparticles align in parallel and the resulting dipolar interactions are sufficiently large to overcome thermal motion and to reorient the magnetic CNTs favouring the formation of chains of aligned carbon nanotubes. These chain-like structures are formed by connecting the magnetic CNTs in line, touching each other in a head to tail fashion - i.e. north to south pole (at positions indicated by arrows in figure $5 \mathrm{c}$ and $\mathrm{d}$ ).

This behaviour has been reported for different magnetic nanoparticles systems. It takes place due to the anisotropic nature of the dipolar interaction. When comes into play, the north and south poles of the dipolar nanomagnets attract each other while particles coming 
close to each other side by side with the magnetization direction parallel will be repelled, thus favoring the formation of nanoparticle chains. [76] Therefore, at first glance one could find astonishing that some magnetic CNTs were found connected in parallel chains (figure $5 b)$. This can be explained by either capillary and van der Waals forces or the fact that long magnetic chains whose end points are not next to each other will also attract each other forming double or triple chains which are parallel and close to each other but are offset to each other along the long axis.

b. Ni/NiO-coated CNTs

In the second strategy designed (figure 3b), the synthesis of well-defined, anisotropic magnetic nanotubes, using again the CNTs as templates, was carried out. Again, the sp ${ }^{2}$ carbon structure was preserved obtaining in this case a ferromagnetic-like behavior at room temperature. $\mathrm{Ni} / \mathrm{NiO}$-coated $\mathrm{CNTs} / \mathrm{Pt}$ nanocomposites were produced exploiting a stepwise reduction of $\mathrm{NiCl}_{2}$ using hydrazine, following a procedure that starts with nanocomposites of CNTs previously functionalized with Pt nanoparticles. [77] With this step in mind, a previous CNTs polyelectrolyte functionalization needs to be carried out using another polyelectrolyte, the polyallylamine hydrochloride (PAH). The advantages in this case stem from the fact that ensure the presence of well-dispersed, individual nanotubes and additionally offers a homogeneous distribution of positive charges. This latter distribution of charges drives Pt nanoparticles once present in solution to be deposited onto CNTs.

These Pt nanoparticles were used as catalytic islands so that, the magnetic material can be grown directly on the CNT outer surface in a process that is mediated by this assembled layer of presynthesized, catalytic Pt nanoparticles. This intermediate step yields organicinorganic hybrid composites that serve as 1D substrates for the preparation of magnetic CNT-supported $\mathrm{Ni} / \mathrm{NiO}$ nanotubes.

Suspensions of Ni nanowires have also been proposed as magneto-optical switches because of their ability to scatter light that is perpendicularly incident to the wire axis. [78] These nanowires, when ferromagnetic, have large remnant magnetization owing to their large aspect ratios (increased contribution of the shape anisotropy) and hence can be used in lowfield environments where superparamagnetic beads do not perform at all. [79] Additionally, since these composite structures involve a $\mathrm{Ni} / \mathrm{NiO}$ antiferromagnetic/ferromagnetic interface, an exchange bias effect is expected which could find promising applications in magnetoresistive devices. [80]

The deposition of a very uniform and homogenous layer of $\mathrm{Ni}$, without surfactants or other stabilizers in water solution represents a relevant issue since their use would induce a perturbation of the magnetic properties displayed. [42, 81]

Figure 6 shows representative TEM and HRTEM images of the samples produced. These images reflect the homogenous coating of individual CNTs and reveal the multidomain and crystalline nature of the $\mathrm{Ni} / \mathrm{NiO}$ layers on the final composite. By Fourier transform analysis (of dark field images, not shown), the following intershell and interplanar distances were determined; MWNTs $(3.36 \AA), \mathrm{Pt}(2.19 \AA)$ and Ni $(2.03 \AA$ and $1.72 \AA)$, corroborating the envisioned structure of $\mathrm{CNTs} @ \mathrm{Pt} / \mathrm{Ni} / \mathrm{NiO}$ nanocomposites. The reader has to take into account that CNTs@Pt/Ni nanocomposites were exposed to an oxygen-rich environment during the magnetic material deposition process (aqueous solution) promoting the formation of the chemically stable $\mathrm{NiO}$ outer layer around each $\mathrm{Ni}$ nanocrystal/nanoshell. [82] This surface passivation process provides the samples with an additional surface stabilization and simultaneously protects the inner metallic Ni from further oxidation. At 
this point it can also be underlined the importance of having a continuous shell of a magnetic material onto the CNTs, contrary to the nanocomposites produced on the first strategy, in which the individuality of the iron oxide nanoparticles was kept once deposited onto the CNTs. This has indeed consequences on the magnetic properties displayed, as detailed later on in this chapter.

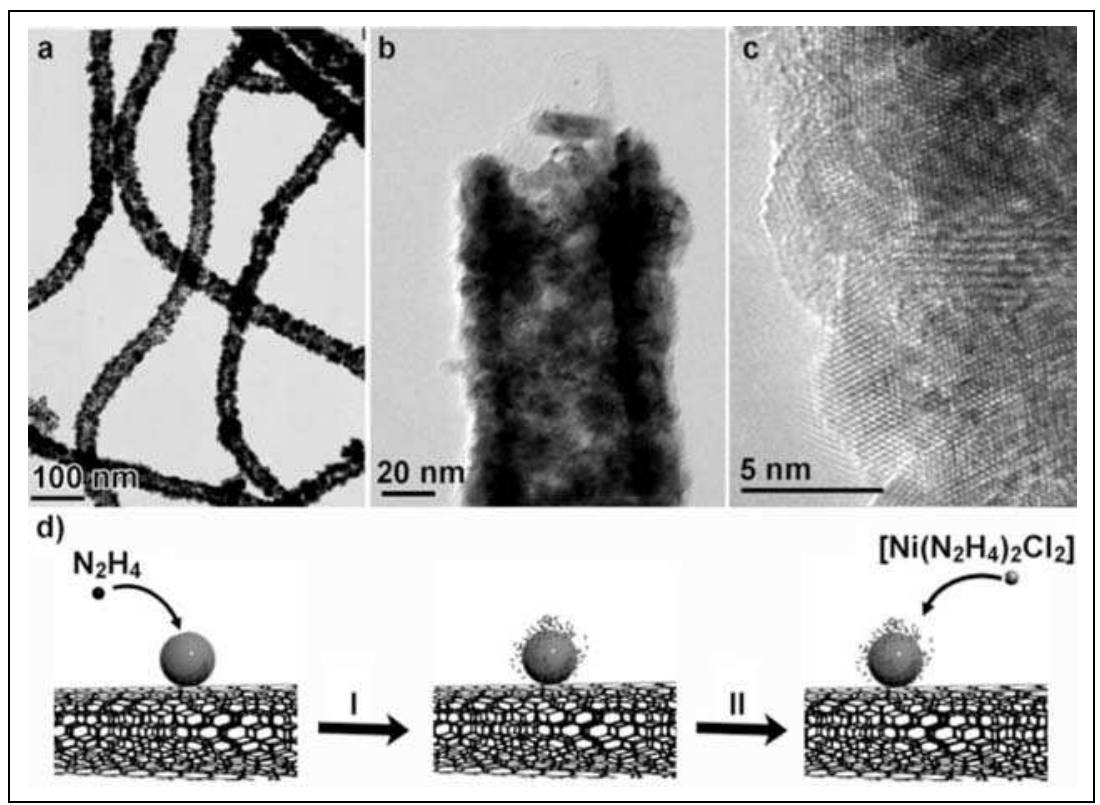

Fig. 6. Representative TEM images of the CNTs@Pt structures coated with a uniform outer layer of $\mathrm{Ni} / \mathrm{NiO}$. HRTEM image reflecting the polycrystalline nature of the $\mathrm{Ni} / \mathrm{NiO}$ layer deposited onto the CNTs@Pt nanocomposites. d) Illustration of Ni reduction on the CNT/Pt side-walls. Step I involves the decomposition of hydrazine on the surface of $\mathrm{Pt}$ nanoparticles, which results in a charged surface, and step II the reduction of the hydrazine/Ni complex on the charged Pt surface.

The catalytic behavior of the small Pt nanoparticles play a crucial role in the formation of the metallic Ni on the walls of the CNTs by catalyzing the reduction of the Ni/hydrazine complex formed in aqueous solution, as described below. This reaction allows the CNT/Pt nanocomposites to be coated with a uniform and homogeneous Ni layer (ca. $10 \mathrm{~nm}$ thick), with no need for surfactants or other stabilizers in aqueous solution. This feature is relevant since the use of surfactants or other stabilizers usually hinders subsequent manipulation and implementation in different applications. The CNT/Pt@Ni composites are stable in solution (most likely because of the presence of a negatively charged $\mathrm{NiO}$ surface layer) and their surface is free of surfactants, thus allowing further functionalization if required. The proposed mechanism for the formation of metallic Ni on the surface of the Pt nanoparticles is depicted schematically in Figure $6 \mathrm{~d}$. Complexes between transition-metal ions and hydrazine are easily formed in water. [83] Such complexes can be decomposed in the presence of hydroxy groups, [84] in a process where $\mathrm{Ni}(\mathrm{II})$ complexes are reduced to $\mathrm{Ni}^{0}$. However, the catalytic decomposition of hydrazine in the presence of $\mathrm{Pt}$ nanoparticles can 
take place on their surface by an electrophilic addition, that is, by forming electrophilic radicals that can then react with other hydrazine molecules from solution. The presence of metallic platinum nanoparticles therefore implies the reduction of nickel complexes without the need for additional hydroxy groups present in solution. In fact, we observed that reduction of $\mathrm{Ni}^{2+}$ did not occur in experiments using PAH-functionalized CNTs in the absence of $\mathrm{Pt}$ nanoparticles. Thus, the mechanism of $\mathrm{Ni}$ nanotube formation can be explained in the following terms: the excess hydrazine that is not complexed with $\mathrm{Ni}^{2+}$ ions can be catalytically decomposed on the surface of the platinum nanoparticles supported on the CNTs. This step generates a charged metallic surface (step I, Figure 6d) which promotes the reduction of the $\mathrm{Ni}(\mathrm{II})$ complex into $\mathrm{Ni}^{0}$ (step II, Figure $6 \mathrm{~d}$ ). Subsequent decomposition of hydrazine on the surface of the reduced metal is facilitated, thus favoring further $\mathrm{Ni}$ reduction and growth of a homogeneous shell. The process terminates when all the $\mathrm{Ni}(\mathrm{II})$ has been reduced. The advantage of this surface-catalyzed reduction of $\mathrm{Ni}$ (II) is the formation of a continuous (though polycrystalline) Ni layer rather than an outer shell composed of nanoparticles, with an envisioned improvement of the resulting magnetic properties. Since the CNT/Pt@Ni nanocomposites formed are exposed to an oxygencontaining environment during the deposition of the magnetic material, a stable $\mathrm{NiO}$ outer layer is expected to form, passivating the $\mathrm{Ni}$ shell and preventing a full oxidation of the magnetic material. 82 However, the presence of nickel oxides on the outer surface of the nickel nanotube could not be confirmed by Fourier transform analysis. To determine whether nickel oxides (and/or hydroxides) were formed on the surface of the CNT/Pt@Ni nanocomposites, the samples were examined by X-ray photoelectron spectroscopy (XPS). Analysis of the $\mathrm{Ni} 2 \mathrm{p}$ peaks revealed a main peak for metallic $\mathrm{Ni}(852.8 \mathrm{eV})$ with contributions from different $\mathrm{Ni}$ oxidation states, presumably corresponding to the binding energies of $\mathrm{NiO}$ and $\mathrm{Ni}(\mathrm{OH})_{2}(854.4$ and $856.5 \mathrm{eV}$, respectively). These main lines are accompanied by satellite lines with binding energies that are $6 \mathrm{eV}$ higher, which suggests the presence of these $\mathrm{Ni}$ oxides at the outer surface of the nickel wires. Apart from passivation, this surface oxidation process leads to ferromagnetic/antiferromagnetic (FM/AFM) interfaces (Ni/NiO), which give rise to an exchange bias effect that increases the potencial applications of these nanocomposites, as seen later in the chapter.

\subsection{Magnetic properties}

\section{a. $\mathrm{Fe}_{3} \mathrm{O}_{4} / \mathrm{Y}-\mathrm{Fe}_{2} \mathrm{O}_{3}$-coated CNTs}

In order to check and understand the influence of using CNTs as supports to generate onedimensional magnetic structures and of the CNTs themselves, the magnetic properties of magnetite/maghemite nanoparticles and the compositess were recorded in a Quantum Design Vibrating Sample Magnetometer (VSM). Figure 7 shows hysteresis curves of both structures, collected at $5 \mathrm{~K}$ and $300 \mathrm{~K}$, that summarize the typical superparamagnetic behavior. Both iron oxide nanoparticles and magnetic CNTs show the same superparamagnetic behavior, i.e. the same coercive field $(\mathrm{Hc}=280$ Oe at $5 \mathrm{~K}$ ) and no remanence or coercitivity at room temperature.

At first sight magnetic CNTs seem to have a larger magnetic moment per gram of sample. The saturation magnetization of the original $\mathrm{Fe}_{3} \mathrm{O}_{4} / \gamma-\mathrm{Fe}_{2} \mathrm{O}_{3}$ nanoparticles, whose relative concentration cannot be quantified due to the similarity of $\gamma-\mathrm{Fe}_{2} \mathrm{O}_{3}$ and $\mathrm{Fe}_{3} \mathrm{O}_{4}$ XRD spectra present a lower value than the bulk magnetization of $\mathrm{\gamma}-\mathrm{Fe}_{2} \mathrm{O}_{3}\left(60-80 \mathrm{Am}^{2} / \mathrm{kg}\right)$. This was reported to be dependent on the particle size and on the different degree of vacancy 
ordering of the particles which is directly related to the sample preparation method.[85] Nevertheless, when assembled onto the surface of MWCNTs the saturation magnetization is increased by approximately $17 \%$ compared to the initial particle powder and agrees rather well with the bulk magnetization of $\gamma_{-}-\mathrm{Fe}_{2} \mathrm{O}_{3}$. The origin of this magnetization increase is not clear and needs further investigation but the presence of nickel traces from the catalytic $\mathrm{Ni}$ surfaces used to grow the CNTs should also be taken into account. The error bar of the sample weight (less than $0.1 \mathrm{mg}$ ) could represent a maximum deviation of $1 \%$ on the magnetization, far away from the obtained $\approx 17 \%$.

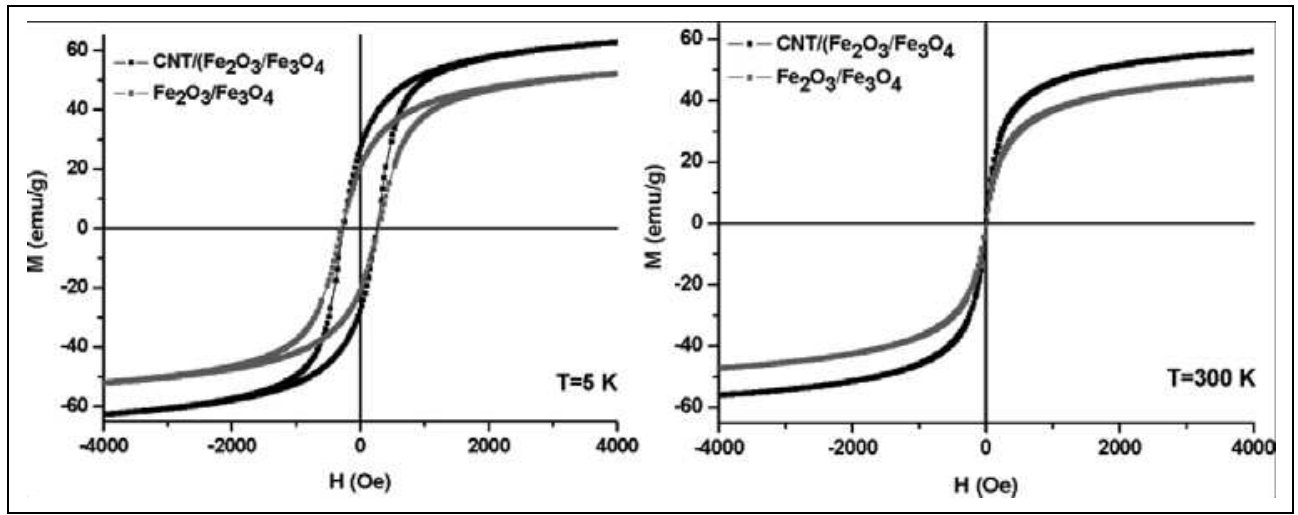

Fig. 7. Magnetic hysteresis cycles for $\gamma-\mathrm{Fe}_{2} \mathrm{O}_{3} / \mathrm{Fe}_{3} \mathrm{O}_{4}$-coated CNTs (black lines) and $\gamma-$ $\mathrm{Fe}_{2} \mathrm{O}_{3} / \mathrm{Fe}_{3} \mathrm{O}_{4}$ nanoparticles (grey lines), showing $\mathrm{M}-\mathrm{H}$ data at 5 (a) and 300 (b) $\mathrm{K}$.

\section{b. Ni/NiO-coated CNTs}

The overall magnetic behaviour of these nanowires, comprising magnetic nanocrystals of $\mathrm{Ni}$ and $\mathrm{NiO}$, is a result of both the properties of the individual constituents (dependent on intrinsic parameters like the size, shape and structure), the morphology they have acquired (anisotropic nanowires) and their interparticle interactions, with a negligible magnetic contribution from the CNTs. Some characteristics of a collective superspin-glass state that can be considered as counterparts of a conventional spin glass are reported, as a consequence of random orientations of the easy axes of the nanocrystals in every wire and the long-range character of the dipolar interactions. In the absence of an external magnetic field the magnetic moment of these nanocrystals constituting the composites would be in a blocked state, unless thermal activation would be able to overcome the anisotropy energy barrier and induce flipping of the moment between easy directions (superparamagnetism). [13] However, these assemblies of nanocrystals forming wires and containing an interface between a ferromagnet $(\mathrm{FM})(\mathrm{Ni})$ and an antiferromagnet (AFM) (NiO) exhibit an additional unidirectional anisotropy due to magnetic coupling at the FM/AFM interface (exchange bias) which leads to magnetization stability. [30]

Exchange biasing has important technological applications, such as in giantmagnetoresistance-based spin valves that are used in hard drive read heads and other spintronics applications.[31] In nanoscale systems, the key factor in this magnetic interaction would be controlling the volume, the shape, crystallinity and structure of both FM and AFM materials. [80, 82, 86-90] Ni/ NiO nanowires offer therefore an attractive approach to control 


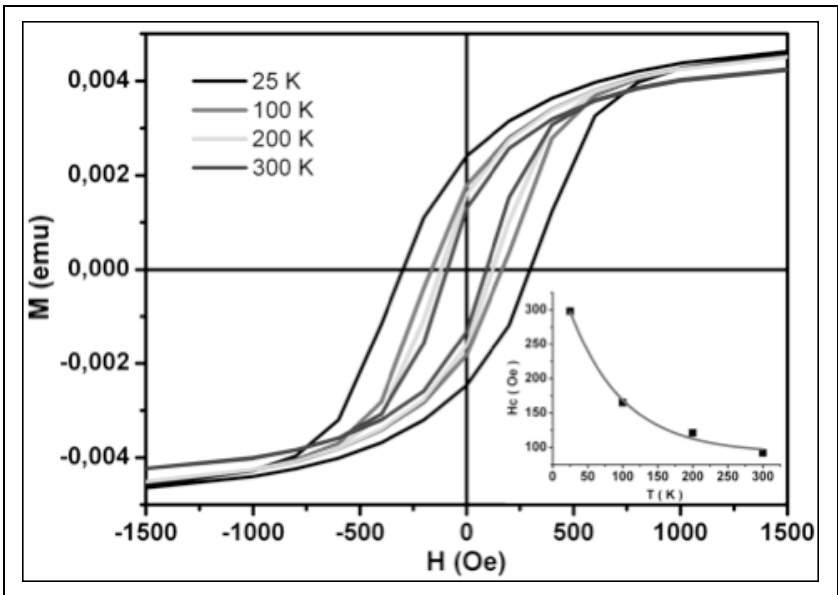

Fig. 8. Hysteresis loops at 25, 100, 200 and $300 \mathrm{~K}$ of Ni/NiO-coated CNTs/Pt nanocomposites. The inset shows the temperature dependence of coercivity $\left(\mathrm{H}_{\mathrm{C}}\right)$.

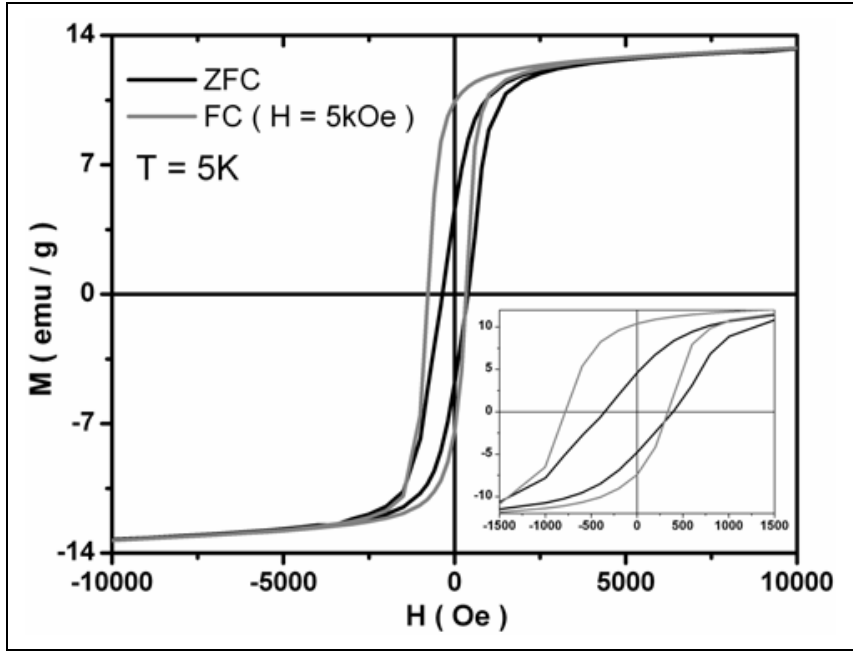

Fig. 9. a) Magnetic hysteresis loops at T1/45 K (zero-field cooled (ZFC) and field cooled (FC) (5 kOe)). b) Magnified view at smaller fields.

exchange coupling, an anisotropic morphology and spin-glass-like behaviour. Significant technological impacts based on this type of nanosized assemblies can thus be expected.

The main results obtained on the magnetic properties of CNTs@Pt/Ni/NiO nanocomposites were also measured in the solid state as powders, but in this case using a superconducting quantum device magnetometer (SQUID). The hysteresis loops, measured at 25, 100, 200 and $300 \mathrm{~K}$ are plotted in figure 8 . The large values of the coercive field, even at room temperature, indicate that the magnetic moments are in a blocked state in the homogeneous $\mathrm{Ni} / \mathrm{NiO}$ shell around every CNT. Each nanowire can be considered as a heterogeneous magnetic system consisting of FM Ni nanocrystals surrounded by AFM $\mathrm{NiO}$ shells, 
homogeneously coating the CNTs/Pt nanotubes. The ferromagnetic component is confirmed by hysteresis (present at $300 \mathrm{~K}$ and lower temperatures), with coercivities $\left(\mathrm{H}_{\mathrm{C}}\right)$ and remnant magnetizations. The antiferromagnetic NiO was identified by XPS (X-ray photoemission spectroscopy) and STEM (scanning transmission electron microscopy) analysis. The oxygen-rich environment of the nanocomposites (in aqueous solution) causes the formation of the stable $\mathrm{NiO}$ outer shells and favors therefore the appearance of interfacial FM/AFM exchange anisotropy. Indeed, when the system is cooled under an applied magnetic field of magnitude $\mathrm{H}_{\mathrm{FC}}=5 \mathrm{kOe}$, the hysteresis loops of the nanocomposites are shifted with respect to the field axis (figure 9), confirming the presence of unidirectional anisotropy due to the exchange coupling at the interface of the FM/AFM materials.[30] Figure 9 shows hysteresis curves collected at $5 \mathrm{~K}$ (zero-field cooled (ZFC) and FC (5kOe)) displaying this shift along the direction of the cooling field with coercivities $\mathrm{H}_{\mathrm{C}}=\left|\mathrm{H}_{\mathrm{C} 1}-\mathrm{H}_{\mathrm{C} 2}\right| / 2$ $=370 \mathrm{Oe}(\mathrm{ZFC})$ and $\mathrm{H}_{\mathrm{C}}=560 \mathrm{Oe}(\mathrm{FC})$ and exchange bias field $\mathrm{H}_{\mathrm{E}}=-\left(\mathrm{H}_{\mathrm{C} 1}+\mathrm{H}_{\mathrm{C} 2}\right) / 2=225 \mathrm{Oe}$ (in the FC curve). The relatively large value of the exchange bias field $\mathrm{H}_{\mathrm{E}}$ indicates an unidirectional exchange anisotropy due to the exchange interaction between the uncompensated surface spins of $\mathrm{NiO}$ and metallic $\mathrm{Ni}$ in the $\mathrm{Ni} / \mathrm{NiO}$-coated CNTs@Pt nanocomposites. This shift of the hysteresis loop can be established either by cooling the FM/AFM material in a magnetic saturation field below the Néel temperature of the AFM (5 $\mathrm{kOe}$ in this case) or by depositing both materials in an external magnetic field.[80]

Exchange bias at FM/AFM interfaces is also characterized by a coercivity enhancement, revealing the induced unidirectional anisotropy. The coercive field $\mathrm{H}_{\mathrm{C}}=90 \mathrm{Oe}$ at $300 \mathrm{~K}$ of the Ni/NiO-coated CNTs/Pt nanocomposites is larger than typical values reported for bulk Ni. This can be explained taking into account an influence of the interface anisotropy, which through exchange coupling can modify the magnetism of the $\mathrm{Ni} / \mathrm{NiO}$ nanocrystals. Roy and co-workers have anticipated that the $\mathrm{NiO}$ shell (in $\mathrm{Ni} / \mathrm{NiO}$ core-shell nanoparticles) can act as a pinning layer, pinning the core spins near the interface of the $\mathrm{Ni}$ inner shell and the $\mathrm{NiO}$ outer shell via exchange interactions. The core spins are thus prevented from rotating freely and follow the applied field, thereby leading to the observed large coercivities.[82] In fact, the loop shifted along the magnetization axis indicates that after the field cooling process a fraction of the uncompensated moments is pinned due to a very high local anisotropy and cannot reverse at the available magnetic field strength. [91]

Summarizing these two first points concerning the magnetic properties of the second type of magnetic nanowires, in both cases there are important contributions from different anisotropies governing different mechanisms. This corresponds to the general contribution dealing with the magnetic anisotropy. This is indeed the quantity that determines the easy magnetization directions of the materials and it is also decisive for the magnetization reversal in external fields. [12]

In both cases the magnetocrystalline anisotropy concerning the crystalline structures of magnetite and maghemite in the first case and metallic $\mathrm{Ni}$, nickel oxide and hydroxides contribute similarly, and there is actually no sense in a comparison as taking into account the nature of different materials.

There are however other three main contributions concerning the shape anisotropy, the surface anisotropy and the unidirectional/uniaxial anisotropy which may become dominant depending on the case, helping to understand therefore the different behavior.

Although having monodimensional nanostructures in both cases, we can consider the discontinuity in the magnetic materials if the outer magnetic shell around the CNTs is formed by individual nanoparticles. On the second case however, as forming the magnetic 
shell by reducing the $\mathrm{Ni}^{2+}$ ions in a step-by-step process, we end up with continuous shells of $\mathrm{Ni}$ (that become $\mathrm{Ni} / \mathrm{NiO}$ ) onto the CNTs. This is not a trivial question when taking into account the shape anisotropy and surface anisotropy contributions, since in the first case we have spherical magnetic nanoparticles (assembled as a cylindrical shell) and cylindrical structures in the second case.

The third and additional contribution is present only in the second case of $\mathrm{Ni} / \mathrm{NiO}$ coated $\mathrm{CNTs} / \mathrm{Pt}$ nanocomposites. Due to the displayed morphology in which a ferromagnetic/ antiferromagnetic interface is established. Thus, these composites were found to exhibit characteristic features of an exchange biased system. By this phenomenon, the magnetic behaviour of the ferromagnetic component in the nanocomposites is markedly affected by the outer shell of $\mathrm{NiO}$, as was reflected by the open hysteresis loops. This interface generates an unidirectional anisotropy which increases the total anisotropy $\mathrm{K}$ so that the thermal energy at room temperature is overcome and the magnetic moments of the composites remain magnetically stable in the time window of the measurement.

\subsubsection{Collective dynamics at low temperatures}

The study of the magnetic properties of these nanoscaled systems of CNTs-based wires emphasize the dominant role of the surface/interface effects on the intrinsic properties of nanoscale systems and the competition with the interactions in the case of assemblies leading to characteristics magnetic behaviors. A further step in these fundamental studies will concern the dynamic aspect of the phenomena.

Magnetization versus temperature measurements at two different magnetic fields $(\mathrm{H}=100$ and $1000 \mathrm{Oe}$ ) for the $\mathrm{Ni} / \mathrm{NiO}-$ coated $\mathrm{CNTs} / \mathrm{Pt}$ are included in figure 10. ZFC and FC magnetization curves split below $\mathrm{T}=300 \mathrm{~K}$ and $\mathrm{T}=100 \mathrm{~K}$ at the applied magnetic fields and in both cases the ZFC magnetization curve exhibits a peak around $\mathrm{T}=40 \mathrm{~K}$. This temperature indicates a collective freezing of magnetic moments (blocking temperature $T_{B}$ ).

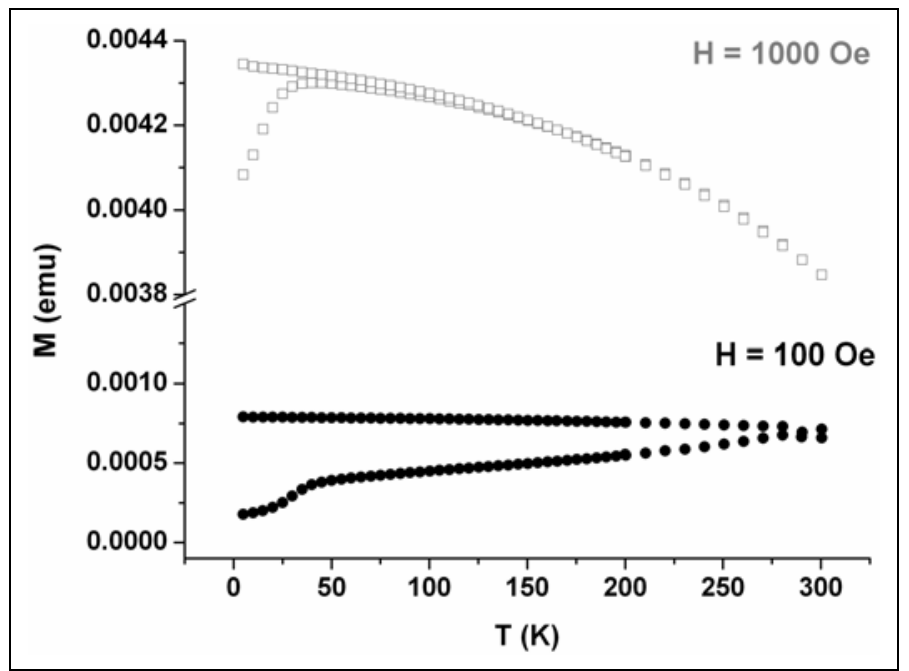

Fig. 10. Field cooled (FC) (5 kOe) and zero-field-cooled (ZFC) magnetization measurements of $\mathrm{Ni} / \mathrm{NiO}$-coated CNTs/Pt nanocomposites. 
The irreversibility shown is strongly dependent on the magnitude of the applied magnetic field and can be presumably associated to slow relaxation processes for an assembly of interacting nanocrystals. This behaviour can be attributed to a random distribution of strong magnetic dipole-dipole interactions and surface anisotropy effects between the Ni cores and the $\mathrm{NiO}$ shells in the nanocrystals. The fact that the temperature at which the irreversibility takes place decreases with increasing magnetic fields [92] can be explained if the effects of the anisotropy field and the dipole-dipole interaction are overcome by the applied magnetic field. [93]

Indeed, one of the most challenging questions in nanoparticulated systems concerns the collective dynamics at low temperatures. In a dilute system, the magnetic dipole-dipole interaction between the particles is negligible compared to the anisotropy energy of an individual nanoparticle. The dynamics follow the predictions of the Néel-Brown model [10] and the system is considered as purely superparamagnetic. However, in a concentrated system the dipole-dipole interaction can be of the order of the particle anisotropy energy and strongly affects the low-temperature dynamics. Three-dimensional random distributions and random orientation of anisotropy axes of such nanoparticles in an insulating matrix with high enough packing density will create a competition of different spin alignments. [94] Despite sophisticated experimental work [95-98] and Monte Carlo simulations [99-101] supporting collective dynamics at low temperature, there are also contradictory results in favor of superparamagnetism behaviour and non-collective blocking. If a low-temperature collective superspin-glass state is formed, typical properties of an ordinary atomic spin glass should be observed in this phase. The collective behaviour of the $\mathrm{Ni} / \mathrm{NiO}$ nanowires is not exclusive of conventional spin glasses and indeed has been reported in systems of concentrated magnetic particles [52] and other nanostructured magnetic materials, where dipolar interactions introduce a collective state and magnetic relaxation dependence. In order to label the nanowires collective behaviour as spin-glass like, we report memory effects by means of a ZFC magnetization experiment with a $2 \mathrm{~h}$ stop during cooling at zero field. [51]

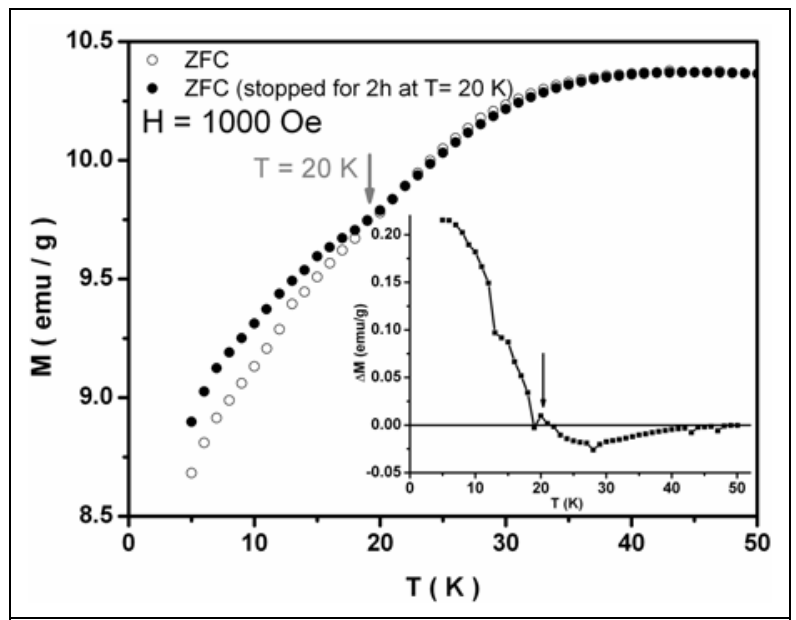

Fig. 11. ZFC magnetization curves measured with and without waiting time $\left(t_{w}=2 h\right)$ showing memory effect. 
Figure 11 illustrates the memory effect of the ZFC magnetization after this stop-and-wait protocol performed below the blocking temperature. The sample was zero field cooled down to $5 \mathrm{~K}$ twice, after a waiting time $t_{w}=2 \mathrm{~h}$ at $20 \mathrm{~K}\left(\mathrm{~T}_{\mathrm{w}}<\mathrm{T}_{\mathrm{B}}=40 \mathrm{~K}\right)$ in the second case. The "stop-and-wait" magnetization data (solid symbols) lie significantly above the conventional ZFC magnetization at temperatures lower than $20 \mathrm{~K}$ (temperature at which the stop-andwait experiment took place). This difference below $\mathrm{T}_{\mathrm{w}}$ indicates that the magnetic moment configuration spontaneously rearranges towards equilibrium as increasing their correlation length. This implies that the correlation between the nanocrystals moments develops in a similar way as the correlation between the spins in spin glasses. Therefore, when interpreting the dynamic behaviour of these interacting $\mathrm{Ni} / \mathrm{NiO}$ nanocrystals in the nanowires, the effects of the spin-glass-like correlations may have to be taken into account. The dynamic magnetic properties observed in the Ni/NiO-coated CNTs/Pt nanocomposites seem to favour the spin-glass hypothesis explaining the observed collectivity and glassiness.

\section{Conclusion}

We have reported the effective magnetic functionalization of CNTs by coating them with iron oxide nanoparticles using the polymer wrapping and $\mathrm{LbL}$ assembly techniques, or in a step-by-step process reducing $\mathrm{Ni}$ on their surface. Upon such functionalization, the alignment of $\mathrm{CNT}$ chains in relatively small external magnetic fields was demonstrated. The resulting magnetic CNTs-based structures are excellent candidates to be used as building blocks for the fabrication of novel composite materials with a preferential orientation of the magnetic CNTs.

\section{Acknowledgments}

The authors acknowledge the financial support of this work under Project MAT2008-06126 by the Spanish Ministerio de Ciencia e Innovación, under project INCITE08PXIB209007PR by the regional government (Xunta de Galicia, Spain) and by the L'Oréal-UNESCO Woman in Science Program. V. S. acknowledges the Ramón y Cajal Program fellowship (Ministerio de Ciencia e Innovación).

\section{References}

[1] Puntes, V. F.; Krishnan, K. M. \& Alivisatos, A. P. (2001). Colloidal nanocrystals shape and size control: The case of Cobalt. Science 291, 2115-2117, ISSN: 0036-8075.

[2] Dumestre, F.; Chaudret, B.; Amiens, C.; Fromen, M.-C.; Casanove, M.-J.; Renaud, P. \& Zurcher, P. (2002) Shape Control of Thermodynamically Stable Cobalt Nanorods throught Organometallic Chemistry, Angew. Chem. Int. Ed. 2002, 41, 4286-4289, ISSN: 0044-8249.

[3] Dumestre, F.; Chaudret, B.; Amiens, C.; Respaud, M.; Fejes, P.; Renaud, P. \& Zurcher, P. (2003). Unprecedented Crystalline Super-Lattices of Monodisperse Cobalt Nanorods. Angew. Chem. Int. Ed. 42, 5213-5216, ISSN: 0044-8249.

[4] Goubault, C.; Leal-Calderon, F.; Viovy, J.-L. \& Bibette, L (2005). Self-Assembled Magnetic Nanowires Made Irreversible by Polymer Bridging. Langmuir, 21, 3725-3729, ISSN: 0743-7463. 
[5] Sellmyer, D. J.; Zheng, M. \& Skomski, R., (2001). Magnetism of Fe, Co and Ni nanowires in self-assembled arrays, J. Phys.: Condens. Matter 13, R433-R460, ISSN: 0053-8984.

[6] Kodama, R. H. (1999). Magnetic nanoparticles. J. Magn. Magn. Mater., 200, 359-372, ISSN: 0304-8853.

[7] Parak, W. J.; Manna, L.; Simel, F. C.; Gerion, D. \& Alivisatos A. P. (2004) Quantum Dots, In: Nanoparticles, Schmid, G. (Ed.), 4-49, Wiley-VCH, ISBN: 3-527-30507-6, Weinheim.

[8] Murray, C. B.; Norris, D. J. \& Bawendi, M. G. (1993). Synthesis and Characterization of Nearly Monodisperse CdE ( $E=$ Sulfur, Selenium, Tellurium) Semiconductor Nanocrystallites. J. Am. Chem. Soc. 115, 8706-8715, ISSN: 0002-7863.

[9] Murray, C. B.; Sun, S.; Doyle, H. \& Betley, T. (2001).Monodisperse 3d transtion-metal (Co, $\mathrm{Ni}, \mathrm{Fe}$ ) nanoparticles and their assembly into nanoparticle superlattices. MRS Bull, 26, 985-991, ISSN: 0883-7694.

[10] Sorensen, C. M. (2001) Magnetism, In: Nanoscale Materials in Chemistry, Klabunde, K. J. (Ed.), 169-221, John Wiley and Sons, ISBN: 0-471-38395-3, New York.

[11] Farle, M. (2003). Magnetic thin films, In: Nanoscale Materials, Liz-Marzán, L. M. \& Kamat, P. (Ed.) 395-422, Kluwer Academic Publishers, ISBN: 1-4020-7366-6, Dordrecht.

[12] Sander, D. (2004). The magnetic anisotropy and spin reorientation of nanostructures and nanoscale films. J. Phys.: Condens. Matter, 16, (R603-636), ISSN: 0053-8984.

[13] Dormann, J. L.; Fiorani, D. \& Tronc, (1997) Magnetic Relaxation in Fine-Particle Systems, In: Advances in Chemical Physics, Prigogine, I. \& Rice, S. A. (Eds.) John Wiley and Sons, 0-471-16285-X, New York.

[14] Gradmann, U., (1993). Magnetism in Ultrathin Transition metal Films, In: Handbook of Magnetic Materials, Buschow, K. H. J. (Ed.) vol. 7, chapter 1, pp 1-96, Elsevier Science, ISBN: 78-4-444-89853-1, Amsterdam.

[15] Eriksson, O., (2001). First principles theory of magnetism for materials with reduced dimensionality, In: Lecture Notes in Physics: Band-Ferromagnetism, Baberschke, K.; Donath, M. \& Nolting, W. (Eds) 243-266, Springer, ISBN: 97-83-5404-2389-8, Berlin.

[16] Lindner, J. \& Farle, M. (2007). Magnetic Anisotropy of Heterostructures, In: Springer Tracks in Modern Physics, 45-96, Springer-Verlag, ISBN: 978-3-540-73462-8-2, Berlin Heidelberg.

[17] Skomski, R. \& Coey J. M. D. (1999) Permanent Magnetism, Institute of Physcis Publishing, ISBN: 0750304782, Bristol.

[18] Blundell, S. (2001). Magnetism in Condensed Matter, Oxford University Press, ISBN: 019 8505922, Oxford.

[19] Burkert, T.; Eriksson, O.; James, P.; Simak, S. I.; Johansson, B. \& Nordström, L. (2004). Calculation of uniaxial magnetic anisotropy energy of tetragonal and trigonal $\mathrm{Fe}$, Co, Ni, Phys. Rev. B, 69, 104426/1-4, ISSN: 0163-1829.

[20] Wu, R. (2001). First principles determination of magnetic anisotropy and magnetostriction in transition metal alloys, In: Lecture Notes in Physics: BandFerromagnetism, Baberschke, K.; Donath, M. \& Nolting, W. (Eds) 46-71, Springer, ISBN: 97-83-5404-2389-8, Berlin. 
[21] Mook, W. M.; Nowak, J. D.; Perrey, C. R.; Carter, C. B.; Mukherjee, R.; Girshick, S. L.; McMurry, P. H. \& Gerberich, W. W. (2007). Compressive Stress Effects on Nanoparticle Modulus and Fracture. Phys. Rev. B 75, 214112/1-4, ISSN: 0163-1829.

[22] Carlton, C. E.; Lourie, O. \& Ferreira, P. J. (2007). In-situ TEM Nano-Indentation of Individual Single-Crystal Nanoparticles, Microsc. Microanal. 13, suppl2, ISSN: 14319276.

[23] Zou, M. \& Yang, D. (2006). Nanoindentation of silica nanoparticles attached to a silicon substrate, Tribology Letters, 22, 189-196, ISSN: 1023-8883.

[24] Rinaldi, A.; Licoccia, S.; Traversa, E.; Sieradzki, K.; Peralta, P.; Dávila-Ibáñez,A. B.; Correa-Duarte, M. A. \& Salgueirino, V. (2009). Novel Plastic Behavior and Superhard of Amorphous and Magnetic Composites Nanostructures, in preparation.

[25] Bin, W.; Heidelberg, A. \& Boland, J. J. (2005). Mechanical properties of ultrahighstrength gold nanowires, Nature Materials, 4, 526-529, ISBN: 1476-1122.

[26] Varghese, B.; Zhang, Y.; Dai, L.; Tan, V. B. C.; Lim, C. T. \& Sow, C.-H. (2008). StructureMechanical Property of Individual Cobalt Oxide Nanowires, Nano Letters, 8, 32263232, ISSN: 1530-6984.

[27] Greer, J. R. \& Nix, W. D. (2006). Nanoscale gold pillars strengthened through dislocation starvation. Phys. Rev. B, 73, 245410/1, ISSN: 0163-1829.

[28] Shan, W.; Mishra, R. K.; Asif, S. A. S.; Warren, O. \& Minor, A. M. (2008). Mechanical annealing and source-limited deformation in submicrometer-diameter Ni crystals, Nature Materials, 7, 115-119, ISBN: 1476-1122.

[29] Cowburn, R. P.; Adeyeye, A. O.; \& Welland, M. E. (1998). Configuration Anisotropy in Nanomagnets. Phys. Rev. Lett. 81, 5414/1-4. ISSN: 0031-9007.

[30] Berkowitz, A. E. \& Takano, K. (1999) Exchange Anisotropy-a review, J. Magn. Magn. Mater. 200, 552-570, ISSN: 0304-8853.

[31] Skumryev, V.; Stoyanov, S.; Zhang, Y.; Hadjipanayis, G.; Givord, D. \& Nogués, J. (2003). Beating the superparamagnetic limit with exchange bias, Nature, 423, 850-853, ISSN: 0028-0836.

[32] Ujfalussy, B.; Szunyogh, L.; Bruno, P. \& Weinberger, P. (1996). First-principles calculation of the anomalous perpendicular anisotropy in a Co monolayer on $\mathrm{Au}$ (111), Phys. Rev. Lett., 77, 1805-1808, ISSN: 0031-9007.

[33] Berkowitz, A. E.; Lahut, J. A. \& VanBuren, C. E. (1980). Properties of Magnetic Fluid Particles, IEEE Trans. Magn., 16, 184-190, ISSN:0018-9464.

[34] Ngo, A. T.; Bonville, P. \& Pileni, M. P. (1999). Nanoparticles of $\mathrm{CoFe}_{2} \mathrm{O}_{4}$ : syntheses and properties, Eur. Phys. J. B., 9, 583-592, ISSN: 1434-6028.

[35] Vestal, C. R., Zhang, Z. J., (2003). Effects of Surface Coordination Chemistry on the Magnetic Properties of $\mathrm{MnFe}_{2} \mathrm{O}_{4}$ Spinel Ferrite Nanoparticles, J. Am. Chem. Soc. 125, 9828-9833, ISSN: 0002-7863.

[36] Shukla, N., Liu, C. Jones, P. M., Weller, D. (2003). FTIR study of surfactant bonding to FePt nanoparticles, J. Magn. Magn. Mater. 2003, 266, 178-184. ISSN: 0304-8853.

[37] L. Néel (1954). Anisotropie Magnétique superficielle et surstructures d'orientation, J. Phys. Radium, 15, 225-239.

[38] Buschow, K. H. J. (1993). Handbook of Magnetic Materials, Elsevier, ISBN: 0444514597, Amsterdam. 
[39] Kodama, R. H.; Berkowitz, A. E.; McNiff Jr., E. J. \& Foner, S. (1996). Surface Spin Disorder in $\mathrm{NiFe}_{2} \mathrm{O}_{4}$ Nanoparticles, Phys. Rev. Lett., 77, 394-397, ISSN: 0031-9007.

[40] Iglesias, O. \& Labarta, A. (2001). Finite-size and surface effects in maghemite nanoparticles: Monte Carlo simulations, Phys. Rev. B, 63, 184416/1-11, ISSN: 01631829.

[41] Dumestre, F.; Martinez, S.; Zitoun, D.; Fromen, M.-C.; Casanove, M.-J.; Lecante, P.; Respaud, M.; Serres, A.; Benfield, R. E.; Amiens, C.; Chaudret, B. (2004) Magnetic nanoparticles through organometallic synthesis: evolution of the magnetic properties from isolated nanoparticles to organized nanostructures, Faraday Discuss., 125, 265-278, ISSN: 0301-7249 .

[42] Respaud, M.; Broto, J.-M.; Rakoto, H.; Fert, A.; Verelst, M.; Snoeck, E.; Lecante, P.; Mosset, A.; Thomas, L.; Barbara, B.; Osuna, J.; Ould Ely, T.; Amiens, C. \& Chaudret, B. (1998). Surface effects on the magnetic properties of ultrafine cobalt particles, Phys. Rev. B, 57, 2925-2935, ISSN: 0163-1829.

[43] Wulfhekel, W.; Zavaliche, F.; Hertel, R.; Bodea, S.; Steierl, G.; Liu, G.; Oepen H. P. \& Kirschner, J. (2003). Growth structure and magnetism of Fe nanostructures on W(001), Phys. Rev. B, 68, 144416/1-9, ISSN: 0163-1829.

[44] Nordblad, P. \& Svedlindh, P. (1998). Spin glasses and Random Fields, In: Spin glasses and Random Fields, Young A. P. (Ed.) 1-27 World Scientific, ISBN: 9810231830, Singapore.

[45] Jonason, K.; Vincent, E.; Hammann, J.; Bouchaud, J. P. \& Nordbald, P. (1998). Memory and Chaos Effects in Spin Glasses, Phys. Rev. Lett. 81, 3243-3246, ISSN: 0031-9007.

[46] Luo, W., Nagel, S. R., Rosenbaum, T. F., Rosensweig, R. E. (1991). Dipole Interactions with random anisotropy in a frozen ferrofluid, Phys. Rev. Lett. 67, 2721-2724, ISSN: 0031-9007.

[47] Sasaki, M.; Jönsson, P. E.; Takayama, H. \& Mamiya, H. (2005) Aging and memory effects in superparamganets and superspin glasses, Phys. Rev. B, 71, 104405/1-9, ISSN: 0163-1829.

[48] Sahoo, S.; Petracic, O.; Kleemann, W.; Nordblad, P.; Cardoso, S. \& Freitas, P. P. (2003). Aging and memory in a superspin glass, Phys. Rev. B 67, 214422-1/5, ISSN: 0163-1829.

[49] Takayama, H. \& Hukushima, K. (2004). Field-Shift Aging Protocol on 3D Ising SpinGlass Model: Dynamical Crossover between the Spin-Glass and Paramagnetic States, J. Phys. Soc. Jpn. 73, 2077-2080, ISSN: 0031-9015.

[50] Yang, H. T.; Hasegawa, D.; Takahashi, M. \& Ogawa, T. (2009). Achieving a noninteracting magnetic nanoparticle system through direct control of interparticle spacing, Appl. Phys. Lett. 94, 013103/1-3, ISSN: 0003-6951.

[51] Rivadulla, F.; López-Quintela, M. A. \& Rivas, J. (2004). Origin of the Glassy magntic Behavior of the Phase Segregated State of the Perovskites, Phys. Rev. Lett. 93, 167206-1/4, ISSN: 0031-9007.

[52] Jonsson, T.; Mattsson, J.; Djurberg, C.; Khan, F. A.; Nordblad, P. \& Svedlindh, P. (1995). Aging in a Magnetic Particle System, Phys. Rev. Lett. 75, 4138-4141, ISSN: 0031-9007.

[53] Hendriksen, S.; Linderoth, S. \& Lindgard, P.-A. (1993) Finite-size modifications of the magnetic properties of clusters, Phys. Rev. B 48, 7259-7273, ISSN: 0163-1829. 
[54] S. Iijima, (1991). Helical microtubules of graphitic carbon, Nature, 354, 56-58, ISSN: 00280836.

[55] Wildoer, J. W. G.; Venema, L. C.; Rinzier, A. G.; Smalley, R. E. \& Dekker, C. (1998). Electronic structure of atomically resolved carbon nanotubes, Nature, 391, 59-62, ISSN: 0028-0836.

[56] Baughman, R. H.; Zakhidov, A. A. \& de Heer, W. A. (2002). Carbon Nanotubes-the Route toward Applications, Science, 297, 787-792, ISSN:0036-8075.

[57] Tomanek, D.; Jorio, A.; Dresselhaus, M. S. \& Dresselhaus, G. (2001). Introduction to the Important and Exciting Aspects of Carbon-Nanotube Science and Technology. In: Carbon Nanotubes Synthesis, Structure, Properties, and Applications, Dresselhaus, M. S. \& Dresselhaus, G. (Eds), Springer, 1-12, ISBN: 3540728643, Berlin Heidelberg.

[58] Correa-Duarte, M. A.; Wagner, N.; Rojas-Chapana, J.; Morsczeck, C.; Thie, M. \& Giersig, M. (2004). Fabrication and Biocompatibility of Carbon Nanotube-based 3D Networks as Scaffolds for Cell Seeding and Growth, Nano Lett. 4, 2233-2236, ISSN: 1530-6984.

[59] Rojas-Chapana, J. A.; Correa-Duarte, M. A.; Ren, Z.; Kempa, K. \& Giersig, M. (2004). Enhanced Introduction of Gold Nanoparticles into Vital Acidothiobacillus ferrooxidans by Carbon Nanotube-based Microwave Electroporation, Nano Lett. 4, 985-988, ISSN: 1530-6984.

[60] White, C. T. \& Todorov, T. N. (1998). Armchair carbon nanotubes as long ballistic conductors, Nature, 393, 240-243, ISSN: 0028-0836.

[61] Wildgoose, G. G.; Banks, C. E. \& Compton, R. G., (2006). Metal Nanoparticles and Related Materials Supported on Carbon Nanotubes: Methods and Applications. Small, 2, 182-193, ISSN: 1613-6810.

[62] Correa-Duarte, M. A. \& Liz-Marzan, L. M. (2006). Carbon Nanotubes as templates for one-dimensional nanoparticle assemblies, J. Mater. Chem. 16, 22-25, ISSN: 1364-5501.

[63] Kamaras, K.; Itkis, M. E.; Hu, H.; Zhao, B. \& Haddon, R. C. (2003). Covalent Bond Formation to a Carbon Nanotube Metal, Science, 301, 1501-1501.

[64] Hirsch, A. (2002). Functionalization of Single-Walled Carbon Nanotubes, Angew. Chem. 41, 1853-1859, ISSN: 0044-8249.

[65] Zhang, Z.; Tang, Z; Kotov, N. A. \& Glotzer, S. C. (2007). Simulations and Analysis of Self-Assembly of CdTe Nanoparticles into Wires and Sheets, Nano Lett., 7,16701675, ISSN: 1530-6984.

[66] Tang, Z.; Wang, Y.; Shanbhang, S.; Giersig, M. \& Kotov, N. A. (2006). Spontaneous Transformation of CdTe Nanoparticles into Angled Te Nanocrystals: From Particles and Rods to Checkmarks, X-Marks, and Other unusual Shapes, J. Am. Chem. Soc., 128, 6730-6736, ISSN: 0002-7863.

[67] Salgueirino-Maceira, V.; Correa-Duarte, M. A.; Hucht, A. \& Farle, M. (2006). Onedimensional assemblies of silica-coated cobalt nanoparticles. Magnetic pearl necklaces. J. Magn. Magn. Mater. 303, 163-166, ISSN: 0304-8853.

[68] Scanna, S. \& Philipse, A. P. (2006). Preparation and Properties of Monodisperse Latex Spheres with Controlled Magnetic Moment for Field-induced Colloidal Crystallization and (bipolar) Chain Formation, Langmuir, 22, 10209-10216, ISSN: 0743-7463. 
[69] Klokkenburg, M.; Dullens, R. P. A.; Kegel, W. K.; Erné, B. H. \& Philipse, A. (2006). Quantitative Real-Space Analysis of Self-Assembled Structures of Magnetic Dipolar Colloids, Phys. Rev. Lett., 96, 037203/1-4, ISSN: 0031-9007.

[70] Correa-Duarte, M. A.; Grzelczak, M.; Salgueirino-Maceira, V.; Giersig, M.; Liz-Marzan, L. M.; Farle, M.; Sierazdki K. \& Diaz, R. (2005). Alignment of Carbon Nanotubes under Low Magnetic Fields through Attachment of Magnetic Nanoparticles, J. Phys. Chem. B, 109, 19060-19063, ISSN: 1520-6106.

[71] Grzelczak, M.; Correa-Duarte, M. A.; Salgueirino-Maceira, V.; Rodrıguez-Gonzalez, B.; Rivas, J. \& Liz-Marzan, L. M. (2007). Pt-Catalyzed Formation of Ni Nanoshells on Carbon Nanotubes, Angew. Chem. Int. Ed., 46, 7026-7030, ISSN: 0044-8249.

[72] Correa-Duarte, M. A.; Sobal, N.; Liz-Marzan, L. M. \& Giersig, M. (2004). Linear Assemblies of Silica-coated gold nanoparticles using carbon nanotubes, Adv. Mater., 16, 2179-2184, ISSN: 0935-9648.

[73] Ostrander, J. W.; Mamedov, A. A. \& Kotov, N. A. (2001). Two modes of Linear Layerby-layer Growth of Nanoparticle-Polyelectrolyte Multilayers and Different Interactions in the Layer-by-layer Deposition, J. Am. Chem. Soc., 123, 1101-1110, ISSN: 0002-7863.

[74] Spasova, M.; Salgueiriño-Maceira, V.; Schlachter, A.; Hilgendorff, M.; Giersig M.; LizMarzan, L. M. \& Farle, M. (2005). Magnetic and Optical tunable microspheres with a magnetit/gold nanoparticle shell, J. Mater. Chem., 15, 2095-2098, ISSN: 1364-5501.

[75] R. Massart (1981). Preparation of aqueous magnetic liquids in alkaline and acidic media, IEEE Trans. Magn. 1981, MAG-17, 1247-1248, ISSN:0018-9464.

[76] K. Butter, P. H. Bomans, P. M. Frederik, G. J. Vroege, A. P. Philipse, A. P. (2003). Direct observation of dipolar chains in ferrofluids in zero field using cryogenic electron microscopy, J. Phys.: Condens. Matter 2003, 15, S1451-S1470, ISSN: 0053-8984.

[77] Ahrenstorf, K.; Albrecht, O.; Heller, H.; Kornowski, A.; GSrlitz, D. \& Weller, H. (2007). Colloidal Synthesis of $\mathrm{Ni}_{x} \mathrm{Pt}_{1-x}$ Nanoparticles with Tuneable Composition and Size, Small, 3, 271-274, ISSN: 1613-6810.

[78] Bentley A. K.; Ellis, A. B.; Lisensky, G. C. \& Crone, W. C. (2005). Suspensions of nickel nanowires as magneto-optical switches, Nanotechnology, 16, 2193-2197, ISSN: 09574484 .

[79] Reich, D. H.; Tanase, M.; Hultgren, A.; Bauer, L. A.; Chen, C. S. \& Meyer, G. J. (2003). Biological applications of multifunctional magnetic nanowires. J. Appl. Phys., 93, 7275-7280, ISSN: 0021-8979.

[80] Fraune, M.; Rudiger, U.; Guntherodt, G.; Cardoso, S. \& Freitas, P. (2000). Size dependence of the Exchange bias field in $\mathrm{NiO} / \mathrm{Ni}$ nanostructures, Appl. Phys. Lett., 77, 3815-3817, ISSN: 0003-6951.

[81] van Leeuwen, D. A.; Van Ruitenbeek, J. M.; de Jongh, L. J.; Ceriotti, A.; Pacchioni, G.; Harberlen, O. D.\& Rosch, N. (1994). Quenching of Magnetic Moments by LigandMetal Interactions in Nanosized Magnetic Metal Clusters, Phys. Rev. Lett., 73, 14321435, ISSN: 0031-9007.

[82] Roy, A.; Srinivas, V.; Ram, S.; de Toro, J. A. \& Mizutani, U. (2005). Structure and magnetic Properties of oxygen-stabilized tetragonal Ni nanoparticles prepared by borohydride reduction method, Phys. Rev. B, 71, 184443/1-10, ISSN: 0163-1829. 
[83] F. Bottomley (1970). The reactions of Hydrazine with Transition-metal Complexes. Q. Rev. Chem. Soc., 24, 617-638, ISSN: 0009-2681.

[84] Wu, S.-H. \& Chen, D.-H. (2004). Synthesis and Stabilization of Ni Nanoparticles in a Pure Aqueous CTAB Solution, Chem. Lett., 33, 406-410, ISSN: 0366-7022.

[85] Morales, M. P.; Veintemillas-Verdaguer, S.; Montero, M. I. \& Serna, C. J. (1999). Surface and Internal Spin Canting in $\gamma-\mathrm{Fe}_{2} \mathrm{O}_{3}$ nanoparticles, Chem. Mater., 11, 3058-3064 , ISSN: 0897-4756.

[86] Makhlouf, S. A.; Parker, F. T.; Spada, F. E. \& Berkowitz, A. E. (1997). Magnetic Anomalies in NiO nanoparticles, J. Appl. Phys., 81, 5561-5564, ISSN: 0021-8979.

[87] Tracy, J. B.; Weiss, D. N.; Dinega, D. P. \& Bawendi, M. G. (2005). Exchange Biasing and Magnetic Properties of Partially and Fully Oxidized Colloidal Cobalt Nanoparticles, Phys. Rev. B, 72, 064404/1-8, ISSN: 0163-1829.

[88] Kodama, R. H.; Makhlouf, S. A. \& Berkowitz, A. E. (1997). Finite Size Effects in Antiferromagnetic NiO Nanoparticles, Phys. Rev. Lett., 79, 1393-1396, ISSN: 00319007.

[89] Spasova, M.; Wiedwald, U.; Farle, M.; Radetic, T.; Dahmen, U.; Hilgendorff, M. \& Giersig, M. (2004). Temperature dependence of Exchange Anisotropy in Monodisperse Cobalt Nanoparticles with a Cobalt Oxide Shell, J. Magn. Magn. Mater., 272-276, 1508-1509, ISSN: 0304-8853.

[90] Salgueirino-Maceira, V.; Spasova, M. \& Farle, M. (2005). Water-Stable, Magnetic SilicaCobalt-Silica Multishell Submicron Spheres, Adv. Funct. Mater., 15, 1036-1040, ISSN: 1616-301X.

[91] Tomou, A.; Gournis, D.; Panagiotopoulos, I.; Huang, Y.; Hadjipanayis, G. C. \& Kooi, B. J. (2006). Weak Ferromagnetism and exchange biasing in cobalt oxide nanoparticle systems, J. Appl. Phys., 99, 123915/1-5, ISSN: 0021-8979

[92] Antoniak, C.; Lindner, J.; Salgueiriño-Maceira, V. \& Farle, M. (2006). Multifrenquency magnetic resonance and blocking behavior of FexPt1-x nanoparticles Phys. Status Solidi (a), 203, 2968-2973, ISSN: 1862-6319.

[93] Zhang, P.; Zuo, F.; Urban III, F. K.; Khabari, A.; Griffiths, P. \& Hosseini-Tehrani, A. (2001). Irreversible Magnetization in Nickel Nanoparticles, J. Magn. Magn. Mater., 225, 337-345, ISSN: 0304-8853.

[94] Brown, Jr. W. F. (1963). Thermal Fluctuations of a Single-Domain Particle, Phys Rev, 130, 1677-1686, ISSN: 0031-9007.

[95] Dormann, J. L.; Fiorani, D.; Cherkaoui, R.; Tronc, E.; Lucari, F.; D’Orazio, F.; Spinu, L.; Nogués, M.; Kachkachi, H. \& Jolivet, J. P., (1999). From pure superparamagnetism to glass collective state in $\gamma-\mathrm{Fe}_{2} \mathrm{O}_{3}$ nanoparticle assemblies, J. Magn. Magn. Mater., 203, 23-27, ISSN: 0304-8853.

[96] Djurberg, C.; Svedlindh, P.; Nordblad, P.; Hansen, M. F.; Bodker, F. \& Morup, S. (1997). Dynamics of an Interacting Particle System: Evidence of Critical Slowing Down, Phys. Rev. Lett., 79, 5154-5157, ISSN: 0031-9007.

[97] Johsson, T.; Svedlindh, P. \& Hansen, M. F. (1998). Static Scaling on an Interacting Magnetic Nanoparticle System, Phys. Rev. Lett., 81, 3976-3979, ISSN: 0031-9007.

[98] Kleeman, W. Petracic, O. Binek, Ch. Kakazei, G. N. Pogorelov, Y. G. Sousa, J. B. Cardoso, S. \& Freitas, P. P. (2001). Interacting ferromagnetic nanoparticles in 
discontinouos $\mathrm{Co} 80 \mathrm{Fe} 30 / \mathrm{Al} 2 \mathrm{O} 3$ multilayers: from superspin glass to reentrant superferromagnetism, Phys. Rev. B, 63, 134423/1-5, ISSN: 0163-1829.

[99] García-Otero, J.; Porto, M.; Rivas, J. \& Bunde, A. (2000). Influence of Dipolar Interaction on Magnetic Properties of Ultrafine Ferromagnetic Particles, Phys. Rev. Lett., 84, 167-170, ISSN: 0031-9007.

[100] Ulrich, M. García-Otero, J. Rivas, J. Bunde, A. (2003). Slow relaxation in ferromagnetic nanoparticles: Indication of spin-glass behavior, Phys. Rev. B, 67, 024416/1-4, ISSN: 0163-1829.

[101] Andersson, J. O.; Djurberg, C.; Jonsson, T., Svedlindh, P. \& Nordblad, P. (1997). Monte Carlo studies of the dynamics of an interacting monodispersive magnetic-particle system, Phys. Rev. B, 56, 13983-13988. ISSN: 0163-1829. 


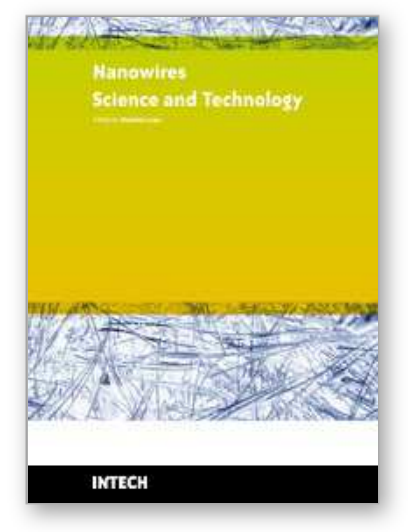

\author{
Nanowires Science and Technology \\ Edited by Nicoleta Lupu
}

ISBN 978-953-7619-89-3

Hard cover, 402 pages

Publisher InTech

Published online 01, February, 2010

Published in print edition February, 2010

This book describes nanowires fabrication and their potential applications, both as standing alone or complementing carbon nanotubes and polymers. Understanding the design and working principles of nanowires described here, requires a multidisciplinary background of physics, chemistry, materials science, electrical and optoelectronics engineering, bioengineering, etc. This book is organized in eighteen chapters. In the first chapters, some considerations concerning the preparation of metallic and semiconductor nanowires are presented. Then, combinations of nanowires and carbon nanotubes are described and their properties connected with possible applications. After that, some polymer nanowires single or complementing metallic nanowires are reported. A new family of nanowires, the photoferroelectric ones, is presented in connection with their possible applications in non-volatile memory devices. Finally, some applications of nanowires in Magnetic Resonance Imaging, photoluminescence, light sensing and field-effect transistors are described. The book offers new insights, solutions and ideas for the design of efficient nanowires and applications. While not pretending to be comprehensive, its wide coverage might be appropriate not only for researchers but also for experienced technical professionals.

\title{
How to reference
}

In order to correctly reference this scholarly work, feel free to copy and paste the following:

Miguel A. Correa-Duarte and Veronica Salgueirino (2010). Magnetic Properties of Nanowires guided by Carbon Nanotubes, Nanowires Science and Technology, Nicoleta Lupu (Ed.), ISBN: 978-953-7619-89-3, InTech, Available from: http://www.intechopen.com/books/nanowires-science-and-technology/magneticproperties-of-nanowires-guided-by-carbon-nanotubes

\section{INTECH}

open science | open minds

\section{InTech Europe}

University Campus STeP Ri

Slavka Krautzeka 83/A

51000 Rijeka, Croatia

Phone: +385 (51) 770447

Fax: +385 (51) 686166

www.intechopen.com

\section{InTech China}

Unit 405, Office Block, Hotel Equatorial Shanghai

No.65, Yan An Road (West), Shanghai, 200040, China

中国上海市延安西路65号上海国际贵都大饭店办公楼405单元

Phone: +86-21-62489820

Fax: +86-21-62489821 
(C) 2010 The Author(s). Licensee IntechOpen. This chapter is distributed under the terms of the Creative Commons Attribution-NonCommercialShareAlike-3.0 License, which permits use, distribution and reproduction for non-commercial purposes, provided the original is properly cited and derivative works building on this content are distributed under the same license. 